Pedro Lazéra Cardoso

\title{
Machine Teaching com Tempo Limitado para \\ Problemas de Regressão
}

Dissertação de Mestrado

Dissertação apresentada como requisito parcial para obtenção do grau de Mestre pelo Programa de Pós-graduação em Informática do Departamento de Informática da PUC-Rio.

Orientador: Prof. Eduardo Sany Laber 
Pedro Lazéra Cardoso

\section{Machine Teaching com Tempo Limitado para \\ Problemas de Regressão}

Dissertação apresentada como requisito parcial para obtenção do grau de Mestre pelo Programa de Pós-graduação em Informática da PUC-Rio. Aprovada pela Comissão Examinadora abaixo:

Prof. Eduardo Sany Laber

Orientador

Pontifícia Universidade Católica do Rio de Janeiro

Prof. Marco Serpa Molinaro

Pontifícia Universidade Católica do Rio de Janeiro

Prof. Hélio Côrtes Vieira Lopes

Pontifícia Universidade Católica do Rio de Janeiro

Rio de Janeiro, 28 de setembro de 2021 
Todos os direitos reservados. É proibida a reprodução total ou parcial do trabalho sem autorização da universidade, do autor e do orientador.

\section{Pedro Lazéra Cardoso}

Graduado em Engenharia de Produção pela Pontifícia Universidade Católica do Rio de Janeiro (2013).

Ficha Catalográfica

Cardoso, Pedro Lazéra

Machine Teaching com Tempo Limitado para Problemas de Regressão / Pedro Lazéra Cardoso; orientador: Eduardo Sany Laber. - Rio de Janeiro : PUC-Rio, Departamento de Informática, 2021.

v., 57 f: il. ; $29,7 \mathrm{~cm}$

1. Dissertação (mestrado) - Pontifícia Universidade Católica do Rio de Janeiro, Departamento de Informática.

Inclui referências bibliográficas.

1. Informática - Dissertação. 2. aprendizado de maquina;. 3. inteligência artificial;. 4. problemas de regressão;. 5. machine teaching.. I. Laber, Eduardo Sany. II. Pontifícia Universidade Católica do Rio de Janeiro. Departamento de Informática. III. Título. 


\section{Agradecimentos}

Ao meu orientador, Eduardo Laber, pela ajuda inestimável e pelas sugestões precisas, sempre mantendo o equilíbrio entre guiar e dar liberdade para o aluno seguir os próprios caminhos. Ao Sérgio, pelo companheirismo durante a pesquisa, em especial pela paciência em rodar os diversos experimentos sem os quais este trabalho não seria possível.

Ao Georges e ao João Guilherme, amigos da vida e do mestrado, e à Clarice, minha namorada, pela parceria em todos os momentos.

Por último, aos meus pais, Alexandre e Eliane, e aos meus irmãos, Juliana e Felipe, pelo apoio incondicional.

O presente trabalho foi realizado com apoio da Coordenação de Aperfeiçoamento de Pessoal de Nível Superior - Brasil (CAPES) - Código de Financiamento 001. 


\section{Resumo}

Cardoso, Pedro Lazéra; Laber, Eduardo Sany. Machine Teaching com Tempo Limitado para Problemas de Regressão. Rio de Janeiro, 2021. 57p. Dissertação de Mestrado - Departamento de Informática, Pontifícia Universidade Católica do Rio de Janeiro.

Este trabalho considera o problema de Regressão com Tempo Limitado. Dados um dataset, um algoritmo de aprendizado (Learner) a ser treinado e um tempo limitado, não sabemos se será possível treinar o modelo com todo o dataset dentro deste tempo. Queremos então elaborar a estratégia que extraia o melhor modelo possível deste algoritmo de aprendizado respeitando o limite de tempo. Uma estratégia consiste em interagir com o Learner de duas formas: enviando exemplos para o Learner treinar e enviando exemplos para o Learner rotular. Nós definimos o que é o problema de Regressão com Tempo Limitado, decompomos o problema de elaborar uma estratégia em subproblemas mais simples e bem definidos, elaboramos uma estratégia natural baseada em escolha aleatória de exemplos e finalmente apresentamos uma estratégia, $T_{W+B H}$, que supera a estratégia natural em experimentos que realizamos com diversos datasets reais.

\section{Palavras-chave}

aprendizado de maquina; inteligência artificial; problemas de regressão; machine teaching. 


\section{Abstract}

Cardoso, Pedro Lazéra; Laber, Eduardo Sany. Limited Time Machine Teaching for Regression Problems. Rio de Janeiro, 2021. 57p. Dissertação de Mestrado - Departamento de Informática, Pontifícia Universidade Católica do Rio de Janeiro.

This work considers the Time-Limited Regression problem. Given a dataset, a learning algorithm (Learner) to be trained and a limited time, we do not know if it's going to be possible to train the model with the entire dataset within this time constraint. We then want to elaborate the strategy that extracts the best possible model from this learning algorithm respecting the time limit. A strategy consists of a series of interactions with the Learner, in two possible ways: sending labeled examples for the Learner to train and sending unlabeled examples for the Learner to classify. We define what the Time-Limited Regression problem is, we decompose the problem of elaborating a strategy into simpler and more well-defined sub-problems, we elaborate a natural strategy based on random choice of examples and finally we present a strategy, $T_{W+B H}$, that performs better than the natural strategy in experiments we have done with several real datasets.

\section{Keywords}

machine learning; artificial intelligence; regression problems; machine teaching. 


\section{Sumário}

1 Introdução $\quad \mathbf{1 0}$

$\begin{array}{lll}1.1 & \text { Definição do Problema } & 11\end{array}$

$\begin{array}{lll}1.2 & \text { Contribuições } & 13\end{array}$

$\begin{array}{lll}1.3 & \text { Organização da Dissertação } & 13\end{array}$

2 Trabalhos Relacionados 14

2.1 Understanding the Role of Adaptivity in Machine Teaching: The Case of Version Space Learners 16

3 Estratégias Propostas $\quad 17$

3.1 Um pseudocódigo para resumir todas as estratégias 17

3.2 Uma estratégia em termos de funções 20

3.3 Esclarecendo algumas premissas sobre o aprendizado 21

3.4 Um paralelo com o aprendizado humano 22

3.5 A estratégia Single Batch 22

3.6 A estratégia Doubling Trick 23

3.7 A estratégia Wrong Probably First (WPF) 26

3.8 A estratégia Wrong Probably First + Best Hypothesis (W+BH) 30

3.9 A estratégia Uncertain Probably First (UPF) 31

3.10 Breve discussão sobre a complexidade de algumas etapas das estratégias 32

3.11 Exemplo da estratégia Wrong Probably First (WPF) 33

4 Avaliação Experimental $\quad \mathbf{3 5}$

4.1 Datasets 35

4.2 Algoritmos de aprendizado 36

4.3 Erro esperado e erro empírico 37

4.4 Medida de qualidade de uma estratégia 38

4.5 Ambiente computacional dos experimentos 39

$\begin{array}{lll}4.6 & \text { Resultados } & 39\end{array}$

4.7 O tamanho do conjunto de treino de cada estratégia 46

4.8 Análise de sensibilidade 48

4.9 Experimentos com a estratégia baseada em incerteza 49

5 Conclusões e Trabalhos Futuros $\quad \mathbf{5 2}$

5.1 Algumas considerações $\quad 52$

5.2 Conclusões 53

Referências Bibliográficas $\quad \mathbf{5 4}$

A Experimentos com outros limites de tempo $\quad \mathbf{5 6}$ 


\section{Lista de Figuras}

3.1 Exemplo com quatro iterações da estratégia $T_{W P F}$.

sample $_{\text {factor }}=4 \quad$ frac $_{\text {start }}=1 / 15 \quad w_{\text {error }}=1 / 3$

4.1 Erro médio (eixo vertical) ao longo do tempo (eixo horizontal) no conjunto de teste para as estratégias $T_{\text {double }}$ e $T_{W+B H}$, com $L=$ Árvores de Regressão. A legenda exibe o erro médio ao fim do experimento.

4.2 Erro médio (eixo vertical) ao longo do tempo (eixo horizontal) no conjunto de teste para as estratégias $T_{\text {double }}$ e $T_{W+B H}$, com $L=$ Random Forest. A legenda exibe o erro médio ao fim do experimento. 43

4.3 Erro médio (eixo vertical) ao longo do tempo (eixo horizontal) no conjunto de teste para as estratégias $T_{\text {double }}$ e $T_{W+B H}$, com $L=$ SVR. A legenda exibe o erro médio ao fim do experimento.

4.4 Erro médio (eixo vertical) ao longo do tempo (eixo horizontal) no conjunto de teste para as estratégias $T_{W+B H}$ e $T_{W P F}$. A legenda exibe o erro médio ao fim do experimento.

4.5 Erro médio (eixo vertical) ao longo do tempo (eixo horizontal) no conjunto de teste para a estratégia $T_{W+B H}$, variando os parâmetro $w_{\text {error }}$ e sample $_{\text {factor }}$. A legenda exibe o erro médio ao fim do experimento.

4.6 Erro médio (eixo vertical) ao longo do tempo (eixo horizontal) no conjunto de teste para as estratégias $T_{U P F}, T_{\text {double }}$ e $T_{W+B H}$, com $L=$ Random Forest. A legenda exibe o erro médio ao fim do experimento.

A.1 Erro médio (eixo vertical) ao longo do tempo (eixo horizontal) no conjunto de teste para as estratégias $T_{\text {double }}$ e $T_{W+B H}$ para $L \in\{$ Árvore de Regressão, Random Forest, SVR $\}$, com o tempolimite alterado para 1 minuto. A legenda exibe o erro médio ao fim do experimento. 


\section{Lista de Tabelas}

4.1 Datasets 36

4.2 Erros dos modelos finais, das estratégias $T_{\text {double }}$ e $T_{W+B H}$. Os valores com fundo cinza mostram que estratégia foi melhor em cada caso (cada par Learner, dataset)

4.3 Tamanho relativo dos modelos finais. Os valores com fundo cinza mostram que estratégia selecionou mais exemplos em cada caso (cada par Learner, dataset)

4.4 Erros dos modelos finais, das estratégias $T_{\text {double }}$ e $T_{U P F}$, para $L=$ Random Forest. Os valores com fundo cinza mostram que estratégia foi melhor em cada caso (cada dataset). A última coluna exibe o tamanho do conj. de treino final de $T_{U P F}$ em relação ao de $T_{\text {double }}$. 


\section{Introdução}

Imagine que queremos treinar uma Árvore de Regressão para rotular posições $^{1}$ de tabuleiros de xadrez. Nesse cenário hipotético, nosso dataset consiste em mais de um bilhão de partidas jogadas online ${ }^{2}$ (das quais extrairemos mais de 50 bilhões de posições, ou exemplos de treinamento) e nosso tempo está restrito a apenas um dia. Apesar de conhecermos um pouco da teoria das Árvores de Regressão, estamos usando uma biblioteca escrita por terceiros e portanto não conhecemos o algoritmo para construção da Árvore ou sua implementação. Considerando a possibilidade de não ser possível rodar o algoritmo em todo o dataset em apenas um dia, como devemos proceder?

Uma abordagem possível é estimar o tamanho da maior subamostra (subconjunto de exemplos) que o algoritmo é capaz de treinar e enviar essa exata quantidade (ou uma quantidade um pouco menor, por segurança) de exemplos para serem treinados. Fica evidente que essa linha não é promissora, uma vez que o tempo total de execução depende do dataset, da implementação do algoritmo de Árvores de Regressão e da configuração da máquina que vai rodar os experimentos. Não bastasse, é possível que o algoritmo tenha um componente aleatório que vai impactar este tempo de execução.

Uma outra abordagem é escolher uma subamostra $S=S_{0}$ muito pequena (por exemplo, de tamanho $\left|S_{0}\right|=1$ ) e repetir os passos abaixo, enquanto houver tempo:

- Treina com a subamostra $S$

- Aumenta o tamanho da subamostra $S$

Essa segunda abordagem resolve nossos problemas mais imediatos:

${ }^{1}$ Não é escopo deste trabalho detalhar como uma Árvore de Regressão avaliaria posições de xadrez. No entanto, convém dar alguma satisfação ao leitor mais curioso. Primeiro, uma posição de um tabuleiro de xadrez deve ser entendida como uma fotografia do tabuleiro (acompanhada da indicação de qual jogador tem a vez). Segundo, tipicamente a avaliação de uma posição de xadrez é associada um número real, cujo sinal indica de quem é a vantagem (positivo = "brancas vencendo", negativo = "pretas vencendo") e cuja magnitude (o valor em módulo) indica o tamanho da vantagem. De forma simplificada, uma avaliação $=-1$, por exemplo, indica que as pretas têm um peão de vantagem.

${ }^{2}$ Em 15/dez/2014, o maior sítio de xadrez online do mundo (chess.com) celebrou a bilionésima partida jogada. Esse número é certamente dezenas de vezes maior atualmente. 
- O algoritmo de aprendizado é uma caixa-preta: não conhecemos a complexidade de tempo do algoritmo, sua implementação ou as configurações da máquina em que rodaremos nosso experimento

- O tempo é um recurso escasso: temos um limite de tempo que talvez impossibilite que rodemos o algoritmo em todo o dataset

- Queremos retornar algum modelo: queremos garantir que ao fim do experimento (dentro do limite de tempo), retornaremos algum modelo de regressão

A priori, em todos os momentos em que aumentamos nossa subamostra na escolha da subamostra inicial e a cada vez que ampliamos o tamanho da subamostra -, escolhemos exemplos do dataset de forma aleatória ${ }^{3}$.

A principal pergunta que este trabalho pretende responder é exatamente essa: dado um algoritmo de aprendizado (cujo funcionamento não conhecemos), um dataset e um tempo limitado para treinamento, podemos fazer melhor do que escolher subamostras de forma aleatória e uniforme?

\section{1}

\section{Definição do Problema}

\subsection{1}

Notação

Antes de enunciar formalmente o problema, vamos definir alguns termos.

- Um exemplo é um vetor $x$ de números reais

$-\mathcal{X}$ é um conjunto de exemplos

- $\mathcal{Y}$ é um conjunto de rótulos possíveis para um exemplo $x \in \mathcal{X}$. Em particular, como estamos tratando de problemas de regressão, assumimos que $\mathcal{Y}$ é um intervalo de $\mathbb{R}$.

- Uma hipótese $h$ é uma função que associa a cada exemplo $x \in \mathcal{X}$ um rótulo $h(x) \in \mathcal{Y}$

- Um dataset é um par $(X, Y)$, onde $X=\left[x_{1}, \cdots, x_{m}\right]$ é um vetor de exemplos com $x_{i} \in \mathcal{X}$ e $Y=\left[y_{1}, \cdots, y_{m}\right]$ é um vetor de rótulos, com $y_{i} \in \mathcal{Y}$

- Um learner $L$ é um algoritmo de aprendizado que recebe um dataset $(X, Y)$ e retorna uma hipótese $h$

\footnotetext{
${ }^{3}$ Abuso de notação: de forma aleatória uniforme, ou seja, cada exemplo tem a mesma chance de ser escolhido
} 
- A classe de hipóteses de um learner $L$ é o conjunto de hipóteses que $L$ é capaz de produzir

Além disso, duas hipóteses $h_{1}$ e $h_{2}$ são distintas (dado um universo de exemplos $D)$ se $h_{1}(x) \neq h_{2}(x)$ para algum $x \in D$

\subsection{2}

\section{0 problema}

Dados um learner $L$, um dataset $(X, Y)$ e um limite de tempo $t_{\text {lim }}$, qual é a estratégia que retorna a melhor hipótese possível (entre as hipóteses que $L$ pode produzir)?

Uma estratégia consiste numa série de interações com $L$, ao fim das quais um modelo de aprendizado treinado (uma hipótese) deve ser retornado. Cada interação pode ser de dois tipos: (i) enviar a $L$ um subconjunto de exemplos rotulados para treinar; (ii) enviar a $L$ um subconjunto de exemplos não-rotulados para medir seu erro nessa amostra.

É importante frisar que a estratégia não tem acesso aos parâmetros que definem o modelo. Se $L$ produzir Árvores de Decisão, por exemplo, isto equivale a dizer que a estratégia não tem acesso aos nós ou à altura de qualquer Árvore retornada por $L$. Em outras palavras, $L$ é uma caixa-preta - tudo que sabemos sobre $L$ é que, ao receber como input uma amostra $\left(X^{\prime}, Y^{\prime}\right)$, este retorna uma hipótese $h$.

\subsection{3}

\section{O paradigma de Machine Teaching}

Machine Teaching estuda a forma mais eficiente de fazer um Learner $L$ aprender uma determinada hipótese [6]. Tradicionalmente, a eficiência é associada ao número de exemplos ou o número de iterações necessários para induzir $L$ a aprender (ou a convergir para) uma hipótese, independente do tempo gasto para realizar a tarefa. Neste trabalho, tratamos do problema de Machine Teaching com tempo limitado: dado um trio (Learner, dataset, $t_{\text {lim }}$ ), talvez não saibamos se é possível treinar $L$ com todo o dataset dentro de $t_{\text {lim }}$. Nesse contexto, a pergunta que queremos responder é a seguinte: qual é a melhor estratégia para treinar $L$ dentro de um determinado tempo? Em resumo, para resolver o problema proposto na seção 1.1.2, trabalhamos com o paradigma de Iterative Machine Teaching ([13], [14]). 


\section{2}

\section{Contribuições}

Este trabalho faz três contribuições: primeiro, define o problema de Iterative Machine Teaching com Tempo Limitado para problemas de Regressão. Apesar de a área de Machine Teaching contar com diversas contribuições sobre induzir Learners a convergirem para uma determinada hipótese, não achamos, entre os trabalhos relacionados, um que trate especificamente do tempo como recurso escasso e principal restrição. Segundo, propõe uma estratégia natural para resolver o problema, $T_{\text {double }}$, baseada na ideia do "doubling trick". Terceiro (e principal), apresenta uma nova estratégia, $T_{W+B H}$, baseada na ideia de dar mais peso a exemplos em que o modelo de aprendizado corrente erra.

Para evidenciar a melhoria que $T_{W+B H}$ apresenta sobre $T_{\text {double }}$, realizamos um conjunto robusto de experimentos, com diversos modelos de aprendizados e datasets reais.

\section{3}

\section{Organização da Dissertação}

Esta dissertação está organizada da seguinte forma: o capítulo 2 trata dos trabalhos relacionados ao tema, em especial os trabalhos no campo de Machine Teaching; o capítulo 3, cerne da dissertação, apresenta as soluções (ou estratégias) desenvolvidas para o problema; o capítulo 4 exibe diversos experimentos para verificar a qualidade das soluções propostas e traz evidências de que nossa estratégia $T_{W+B H}$ tem desempenho superior à estratégia que seleciona exemplos de forma aleatória uniforme; finalmente, o capítulo 5 apresenta conclusões, limitações e possíveis caminhos que este trabalho pode seguir. 


\section{2 \\ Trabalhos Relacionados}

Em [8], o autor categoriza a literatura sobre Machine Teaching por meio duas dimensões: se o Learner sempre formula hipóteses consistentes com todos os exemplos do conjunto de treinamento e o nível de informação que o Teacher tem sobre o Learner. Neste trabalho, não assumimos a consistência das hipóteses ou qualquer conhecimento do Teacher sobre o Learner.

Em [13], o paradigma de Machine Teaching é introduzido, junto com a ideia de um protocolo de comunicação entre o Teacher e o Learner, duas abordagens que adotamos neste trabalho. No artigo, no entanto, sempre se assume que $T^{1}$ tem algum conhecimento sobre $L^{2}$. Em [14] o autor abandona essa restrição de conhecimento, mas é mais restritivo sobre as interações entre $T$ e $L$ : a cada interação, $T$ deve enviar apenas mais um exemplo rotulado para $L$ atualizar seu modelo de aprendizado.

Independente do que é assumido sobre a consistência do Learner ou o conhecimento do Teacher, a maioria dos estudos em Machine Teaching procura minimizar a quantidade de exemplos necessários para fazer o Learner convergir ([8], [6], [19]), provavelmente muito influenciados pelo trabalho de [10]. Em vez de minimizar o número de exemplos treinados, estamos interessados no melhor modelo que conseguimos fazer $L$ retornar dentro de um tempo limitado.

Em [9] o autor aborda o objetivo de Machine Teaching de maneira mais geral, assumindo que o Teacher interage com o Learner para minizar o Learning Cost, que pode ser tanto o número de exemplos utilizados quanto o tempo gasto no processo. Além disso, propõe uma estratégia de seleção de exemplos que escolhe os exemplos em ordem decrescente de erro. Neste trabalho, propomos parametrizar a importância que se dá a escolher exemplos com maior erro de previsão, uma vez que essa abordagem faz com que o conjunto de treino selecionado tenha sua distribuição diferente da distribuição real dos dados.

Stochastic Gradient Descent (SGD) tem sido apontada como uma solução promissora para problemas de aprendizado de máquina supervisionado em larga escala — isto é, problemas em que não há tempo para treinar com todos

\footnotetext{
${ }^{1}$ Lembrete: $T$ é sinônimo de Teacher e de estratégia

${ }^{2}$ Lembrete: $L$ é sinônimo de Learner e de algoritmo de aprendizado
} 
os exemplos rotulados disponíveis [2]. No contexto geral em que a técnica é aplicada [1], se assume que $L$ é um algoritmo online paramétrico, definido por um vetor de parâmetros $w$, ao qual é possível associar uma função $J(w)$ que calcula a perda (erro esperado, erro de generalização) do modelo definido por $w$. Além disso, essa função deve ser diferenciável, a não ser por um conjunto de pontos com probabilidade zero de ocorrer. Aqui, por "solução promissora" queremos dizer "solução que faz $w$ convergir rapidamente para um um valor ótimo ou próximo do ótimo". Sob essas premissas (algoritmo de aprendizado online e paramétrico, função de perda diferenciável), podemos enxergar SGD como uma estratégia de seleção de exemplos. No entanto, as estratégias que propomos nessa dissertação são mais gerais, pois fazem menos exigências sobre $L$. Para $L=$ Árvore de Regressão, um dos algoritmos utilizados na parte experimental desse trabalho, não é óbvio como poderíamos usar a técnica de SGD.

O paradigma de regressão com tempo limitado também tem relação com o paradigma de Active Learning. Em Active Learning, exemplos não rotulados são abundantes, ao passo que obter exemplos rotulados é custoso. O próprio learner $L$ influencia que exemplos serão rotulados. A primeira questão é portanto como elaborar uma estratégia para determinar que exemplos rotular [7] — exemplos esses que serão então usados para treinar $L$. Temos também um problema de seleção de exemplos. A diferença essencial entre os paradigmas é que, ao simular o contexto de Active Learning num problema de regressão com tempo limitado, desprezamos a informação do rótulo correto de cada exemplo na fase de seleção de exemplos. Estamos abrindo mão de uma informação provavelmente e intuitivamente valiosa. Em [12], o autor sugere usar modelos distintos para as fases de selecionar exemplos e de treinar exemplos, para tratar do caso em que usar o modelo principal (o de treino) na fase de seleção de exemplos pode também ser custoso demais. Uma das estratégias propostas nesse artigo, $T_{U P F}$, é baseada no paradigma de Active Learning.

Muitos trabalhos demonstram resultados teóricos sem aplicação prática imediata ao problema tratado nesta dissertação. Ainda que se considere este distanciamento, estes resultados são inspiradores ao confirmarem - mesmo que num contexto simplificado, em que o tempo de processamento não é levado em conta ou em que $T$ tem total conhecimento e ingerência sobre os parâmetros de $L$ - o que tomamos a liberdade de chamar de intuição ou de bom senso. Como exemplo, descrevemos abaixo [5], em que é demonstrado que, quando o objetivo é fazer $L$ convergir mais rápido (treinando com menos exemplos) para uma hipótese $h^{*}, T$ se beneficia ao interagir (testar $L$ ao longo do processo) com $L$. 


\section{1}

\section{Understanding the Role of Adaptivity in Machine Teaching: The Case of Version Space Learners}

No trabalho, um Version Space induzido por uma amostra (subconjunto de exemplos de um dataset) $Z$ é o conjunto de hipóteses consistentes com $Z$. Quando $L$ recebe $Z$ para treinar, $L$ retorna, entre as hipóteses em Ver sion $\operatorname{Space}(Z)$, uma de suas hipóteses preferidas (não necessariamente de forma aleatória).

A preferência (de $L$ entre duas hipóteses $h_{1}, h_{2}$ ) é definida por uma função de afinidade $O M E G A: H \times H \rightarrow \mathcal{R}$. A função recebe um par de hipóteses porque a afinidade de $L$ com uma hipótese $h$ depende de que hipótese $h_{\text {curr }}$ está atualmente configurada em $L$. Em outras palavras, dada uma hipótese corrente $h_{\text {curr }}$ e duas hipóteses $h_{1}, h_{2}$, temos que $L$ prefere $h_{1}$ a $h_{2}$ se $O M E G A\left(h_{\text {curr }}, h_{1}\right)>O M E G A\left(h_{\text {curr }}, h_{2}\right)$.

Um teacher $T$ é uma entidade (uma estratégia) que tenta conduzir $L$ a uma hipótese-alvo $h^{*}$. Para realizar essa condução, a cada instante $t$ a estratégia $T$ seleciona um exemplo $z_{t}$ e o envia a $L$. O ensino termina quando $L$ retorna $h^{*}$.

$T$ é (por definição) adaptativo se considerar $h_{\text {curr }}$ para escolher $z_{t}$, e $T$ é não-adaptativo se ignorar isso.

O trabalho assume que $T$ conhece a função de preferência de $L, O M E G A$ : $H \times H \rightarrow \mathbb{R}$.

Por fim, é demonstrado que para algumas funções de preferência um teacher adaptativo é melhor (precisa de menos iterações ou de menos exemplos) do que um teacher não-adaptativo. Uma outra maneira de dizer isso é que, em alguns casos, podemos fazer melhor do que escolher exemplos de forma aleatória para fazer $L$ aprender. 


\section{3}

\section{Estratégias Propostas}

O cerne desse trabalho é elaborar estratégias de seleção de exemplos (de um conjunto dado de exemplos, possivelmente grande demais para ser treinado dentro de um limite de tempo) para treinar modelos de regressão. Uma exigência a respeito da estratégia é que esta não dependa de um algoritmo de aprendizado específico, uma vez que o algoritmo será entendido como uma entidade que recebe um subconjunto de exemplos rotulados e retorna um modelo treinado. Em outras palavras, uma das exigências é que a estratégia trate $L$ como uma caixa-preta ${ }^{1}$.

Todas as estratégias propostas neste trabalho podem ser resumidas, em alto nível, pelas etapas listadas abaixo:

1. Faz um pré-processamento do dataset

2. Escolhe uma amostra inicial

3. Treina $L$ com esta amostra inicial

4. Enquanto houver tempo, repete:

(a) avalia o modelo corrente (não há treinamento nesta etapa)

(b) seleciona um novo conjunto de exemplos para treino com base nos resultados da avaliação

(c) treina $L$ com o novo conjunto de exemplos

Neste esquema, "avaliar" equivale a avaliar a hipótese corrente (o modelo treinado corrente) de $L$ numa amostra do dataset.

\section{1}

\section{Um pseudocódigo para resumir todas as estratégias}

Este esquema, em pseudocódigo um pouco menos abstrato, está detalhado abaixo. O significado do input segue aquilo definido na seção 1.1.1: $T$ é

\footnotetext{
${ }^{1} \mathrm{Um}$ black box learner
} 
uma estratégia de seleção de exemplos (ou Teacher), L é um algoritmo de aprendizado, $(X, Y)$ é um dataset e $t_{l i m}$ é o limite de tempo. Finalmente, a variável $m=|X|=|Y|$ representa o tamanho (a quantidade de exemplos) do dataset $^{2}$. 


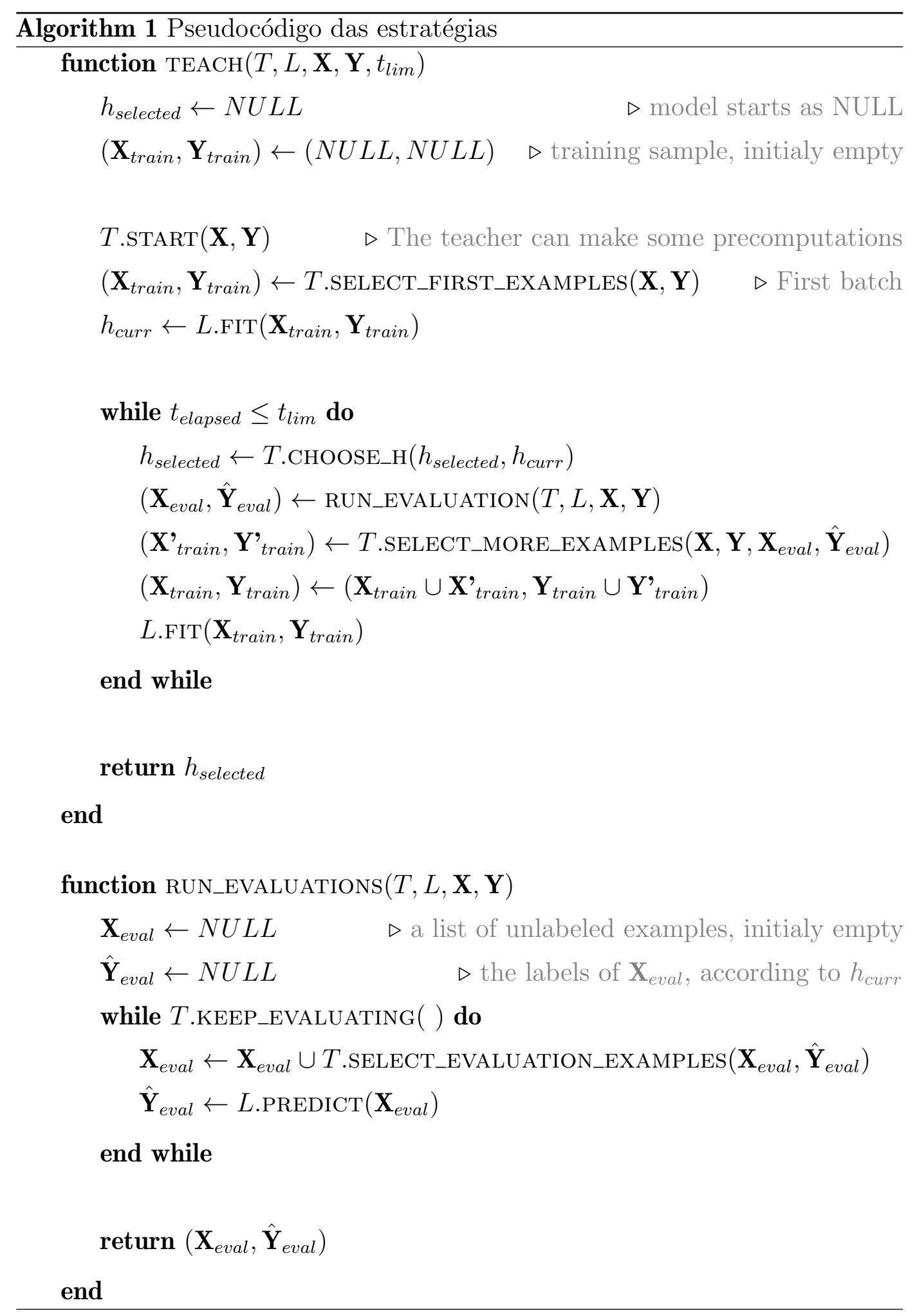


As instruções L.FIT e L.PREDICT indicam respectivamente que o Learner irá treinar com um subconjunto de treino e rotular um subconjunto de exemplos (este último sem os rótulos verdadeiros de cada exemplo).

As variáveis em negrito (como $\mathbf{X}, \mathbf{Y}_{\text {eval }}$ ) representam coleções, como vetores ou conjuntos (estruturas de dados das quais podemos enumerar objetos).

As variáveis sobrescritas com o sinal "^" — o acento circunflexo, ou chapéu, como em $\hat{\mathbf{Y}}_{\text {eval }}$ - representam previsões feitas por algum modelo de aprendizado treinado.

Todas as variáveis globais (variáveis que não estão no input da função e que não são inicializadas dentro da função) são listadas no início do pseudocódigo ${ }^{3}$.

Finalmente, expressões como

\section{$[\mathbf{X}[i]$ for $i$ in $\mathbf{S}]$}

representam um vetor cujo $j$-ésimo elemento vale $X[S[j]]$.

\section{2}

\section{Uma estratégia em termos de funções}

Definir as estratégia de seleção de exemplos abordadas neste trabalho consiste apenas em definir o comportamento (a implementação) de algumas funções:

- START: $(X, Y) \rightarrow N U L L$

- SELECT_FIRST_EXAMPLES: $(X, Y) \rightarrow\left(X^{\prime}, Y^{\prime}\right)$

- SELECT_MORE_EXAMPLES: $\left(X_{1}, Y_{1}, X_{2}, Y_{2}\right) \rightarrow\left(X^{\prime}, Y^{\prime}\right)$

- KEEP_EVALUATING: ()$\rightarrow\{$ true, false $\}$

- SELECT_EVALUATION_EXAMPLES: $(X, Y) \rightarrow X^{\prime}$

- CHOOSE_H: $\left(h_{1}, h_{2}\right) \rightarrow h$

Estas são todas as funções associadas à $T$ no pseudocódigo geral da seção 3.1. Seguindo uma linha de Orientação a Objeto, são as funções com o prefixo "T."

A função T.ST ART $(\mathbf{X}, \mathbf{Y})$ indica um pré-processamento da estratégia $T$ sobre o dataset $(\mathbf{X}, \mathbf{Y})$.

A função T.SELECT_FIRST_EXAMPLES(X,Y) indica o primeiro subconjunto de exemplos selecionado pela estratégia $T$ para treinar $L$. Nesse ponto, ainda não existe hipótese retornada por $L$ (a variável $h_{\text {selected }}$ vale NULL).

${ }^{3}$ Esse caso só ocorre mais para frente 
A função T.SELECT_MORE_EXAMPLES(X, Y, $\left.\mathbf{X}_{\text {eval }}, \hat{\mathbf{Y}}_{\text {eval }}\right)$ indica os subconjuntos subsequentes de exemplos (além do primeiro subconjunto) selecionado pela estratégia $T$ para treinar $L$. Nesse ponto, já existe hipótese retornada por $L$, e portanto esses subconjuntos posteriores são escolhidos com base nos exemplos disponíveis $(\mathbf{X}, \mathbf{Y})$ e também numa avaliação da hipótese corrente sobre uma amostra do dataset $\left(\mathbf{X}_{\text {eval }}, \hat{\mathbf{Y}}_{\text {eval }}\right)$.

As funções T.SELECT_EVALUATION_EXAMPLES $\left(\mathbf{X}_{\text {eval }}, \hat{\mathbf{Y}}_{\text {eval }}\right)$ e T.KEEP_EV ALU ATING( ) indicam a avaliação que $T$ faz sobre a hipótese corrente. Nesse conjunto de interações entre $T$ e $L$, a estratégia $T$ seleciona exemplos apenas para $L$ rotular (prever).

Finalmente, a função $C H O O S E_{-} H\left(h_{\text {selected }}, h_{\text {curr }}\right)$ indica se $T$ vai trocar ou não a hipótese a ser retornada ao fim do experimento.

\section{3}

\section{Esclarecendo algumas premissas sobre o aprendizado}

A respeito do pseudocódigo geral em 3.1, fazemos alguns esclarecimentos: primeiro, $X_{\text {train }}$ e $X_{\text {eval }}$ são subsequências de $X$; segundo, $Y_{\text {train }}$ é o vetor de rótulos (verdadeiros) ${ }^{4}$ dos exemplos em $X_{\text {train }}$; terceiro, $\hat{Y}_{\text {eval }}$ não é o vetor de rótulos corretos de $X_{\text {eval }}$, mas sim o vetor de rótulos previstos pelo modelo corrente; quarto, $h_{\text {selected }}$ e $h_{\text {curr }}$ representam modelos já treinados ${ }^{5}$, diferente de $L$, que é o algoritmo que retorna modelos treinados.

Um aspecto bem mais relevante está implícito no comando abaixo:

$$
\left(\mathbf{X}_{\text {train }}, \mathbf{Y}_{\text {train }}\right) \leftarrow\left(\mathbf{X}_{\text {train }} \cup \mathbf{X}_{\text {train }}^{\prime}, \mathbf{Y}_{\text {train }} \cup \mathbf{Y}_{\text {train }}^{\prime}\right)
$$

Note que estamos assumindo que, a cada iteração, a estratégia aumenta a amostra de treino, ou seja, a nova amostra é a união entre a amostra antiga e alguns novos exemplos. A definição do problema não exige isso - as estratégias em geral podem, a cada rodada, selecionar um conjunto de exemplos completamente distinto do conjunto anterior. Sendo ainda mais puristas, a definição do problema sequer exige que existam rodadas de seleção de exemplo.

Finalmente, outro aspecto que segue do pressuposto acima e que deve ser ratificado está no comando

$$
\operatorname{L.FIT}\left(\mathbf{X}_{\text {train }}, \mathbf{Y}_{\text {train }}\right)
$$

${ }^{4} \mathrm{O}$ gabarito de $X_{\text {train }}$

${ }^{5} \mathrm{O}$ termo "modelo de aprendizado" é usado para denotar tanto um modelo treinado (por exemplo, uma árvore de classificação com nós definidos) quanto o algoritmo que monta modelos treinados (por exemplo, o algoritmo que recebe um conjunto de exemplos e retorna uma árvore treinada) 
Esse linha implica que o algoritmo de aprendizado não é online, ou seja, o modelo corrente não é o modelo anterior modificado pelos novos exemplos selecionados na última rodada - o modelo corrente é o resultado do treinamento de $\left(X_{\text {train }}, Y_{\text {train }}\right)$, e seus parâmetros não dependem de treinamentos anteriores.

Sobre essa premissa, é muito simples adaptar o pseudocódigo (e os códigos) para considerar algoritmos de aprendizado online. Basta alterar a instrução L.FIT $\left(\mathbf{X}_{\text {train }}, \mathbf{Y}_{\text {train }}\right)$ para chamar L.FIT apenas sobre o conjunto incremental $\left(X_{\text {train }}^{\prime}, Y_{\text {train }}^{\prime}\right)$.

\section{4}

\section{Um paralelo com o aprendizado humano}

Para entender a motivação das estratégias propostas nesse trabalho, um paralelo pode ser feito com um professor que quer ensinar um aluno apenas por meio de exemplos.

Primeiro, antes de interagir (por meio de exemplos) com o aluno, o professor recebe a matéria (dataset ou conceito) que deve ser ensinada.

Dada uma matéria, o professor escolhe alguns exemplos rotulados e exibe ao aluno.

Em seguida, enquanto ainda restam dias de aula, o professor interage com o aluno da seguinte forma: (i) aplica uma série (de zero ou mais) testes para o aluno e (ii) com base no resultado do aluno nos testes aplicados, escolhe mais um pedaço da matéria (mais exemplos rotulados) para ensinar ao aluno.

Desviando um pouco do foco deste trabalho, é instigante pensar na tarefa de elaborar uma estratégia (um programa de computador) que um aluno (humano) não consiga diferenciar de um professor (humano também). Isto é, uma estratégia que escolhe exemplos de forma semelhante à forma que um professor humano escolheria ${ }^{6}$.

\section{5}

\section{A estratégia Single Batch}

A estratégia talvez mais simples de todas é aquela que escolhe como subamostra inicial todo o dataset.

\footnotetext{
${ }^{6}$ Fica a cargo do leitor decidir se é assustador ou animador a semelhança entre o pseudocódigo de uma estratégia e o que um professor-humano de fato faria
} 


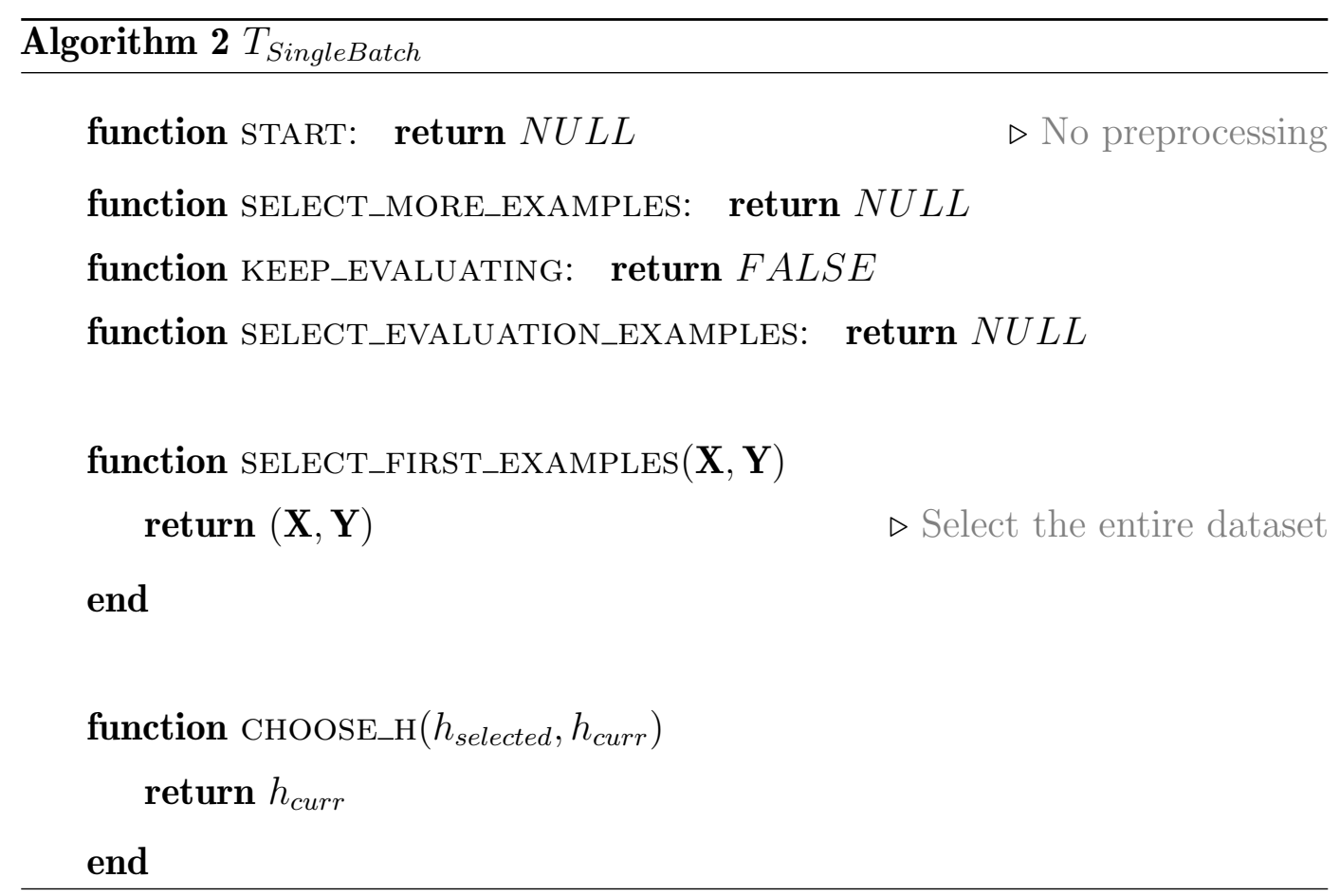

A função SELECT_FIRST_EXAMPLES retorna todo o dataset. Já as funções relativa a avaliações da hipótese corrente não fazem nenhuma avaliação. Finalmente, a função sobre trocar ou não trocar o valor de $h_{\text {selected }}$ sempre seleciona a última hipótese treinada.

O único (enorme) problema desta estratégia é também o motivo pelo qual essa dissertação está sendo escrita: existe a possibilidade de não ser possível treinar com todo o dataset dentro do limite de tempo, caso em que $h_{\text {selected }}=N U L L$.

$T_{\text {SingleBatch }}$ irá funcionar como uma base de comparação para as demais estratégias. Com tempo disponível, nossa escolha natural seria treinar com todo o dataset. Não havendo tempo, queremos saber quão perto chegamos desse cenário ideal, e a medida de proximidade, a ser detalhada no capítulo 4, será a relação entre (i) o erro da hipótese retornada por $T_{\text {SingleBatch }}$ e (ii) o erro da hipótese retornada pela estratégia cuja qualidade queremos medir.

\section{6}

\section{A estratégia Doubling Trick}

Para remediar o problema de $T_{\text {SingleBatch }}$, a estratégia $T_{\text {double }}$ (é como chamaremos "Doubling Trick") começa com uma subamostra inicial pequena ${ }^{7} \mathrm{e}$ vai dobrando seu tamanho enquanto enquanto houver tempo.

\footnotetext{
${ }^{7}$ Pequeno o suficiente para que $L$ treine com uma subamostra deste tamanho dentro do limite de tempo
} 
A fim de controlar o tamanho da amostra inicial, utilizamos o parâmetro frac $_{\text {start }} \in(0,1]$, que representa a proporção de exemplos na amostra inicial. Caso o usuário não informe um valor para o parâmetro, assume-se que frac $_{\text {start }}=\frac{1}{m}$.

De forma muito abstrata, na primeira iteração $T_{\text {double }}$ seleciona $m \cdot$ frac $_{\text {start }}$ exemplos aleatoriamente. A cada próxima iteração $i+1, T_{\text {double }}$ seleciona aleatoriamente mais $\left|S_{i}\right|$ novos exemplos, onde $S_{i}$ é o conjunto de exemplos treinados na iteração $i$.

O pseudocódigo abaixo representa de forma menos abstrata a estratégia $T_{\text {double. }}$. Neste esquema, $\mathbf{v}_{\text {free }_{i d s}}$ representa o conjunto de índices dos exemplos que ainda não foram selecionados para compor a amostra de treino. 


$\underline{\text { Algorithm } 3 T_{\text {double }}}$

function KEEP_EVALUATING: same as in $T_{\text {SingleBatch }}$

function SELECT_EVALUATION_EXAMPLES: same as in $T_{\text {SingleBatch }}$

function CHOOSE_H: same as in $T_{\text {SingleBatch }}$

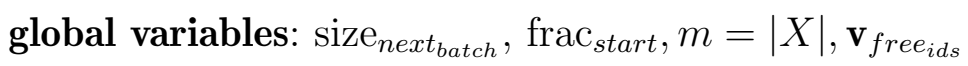
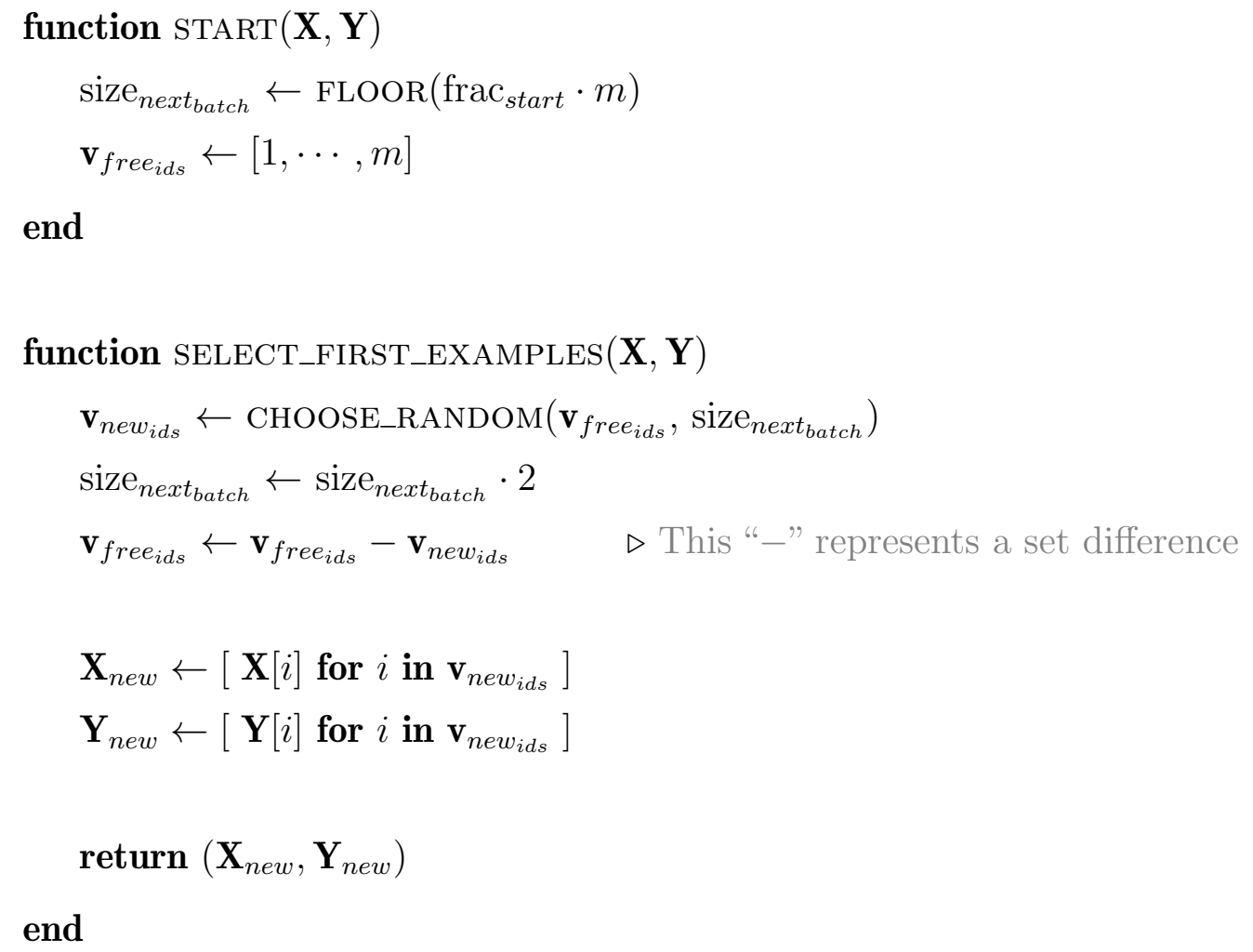

function SEleCt_MORE_EXAmPles $\left(\mathbf{X}, \mathbf{Y}, \mathbf{X}_{\text {eval }}, \hat{\mathbf{Y}}_{\text {eval }}\right)$ return SELECT_FIRST_EXAMPLES $(\mathbf{X}, \mathbf{Y})$

end

Fazemos alguns esclarecimentos para o leitor que mentalmente compila pseudocódigos ${ }^{8}$. Primeiro, o pseudocódigo de $T_{\text {double }}$ não lida com casos de fronteira, como quando size next_batch $>|X|$. Segundo, a chamada de função CHOOSE_RANDOM $(v, k)$, sobre um vetor $v$ e um inteiro positivo $k \leq|v|$, faz um sorteio aleatório uniforme sem reposição ${ }^{9}$ de $k$ elementos de $v$.

${ }^{8} \mathrm{E}$ que não consegue prosseguir a leitura enquanto o código não compilar sem erros em sua cabeça

${ }^{9}$ Nenhum elemento será escolhido duas vezes 
Finalmente, destacamos que a escolha de novos exemplos é idêntica à escolha dos primeiros exemplos. Isto porque $T_{\text {double }}$ não avalia a hipótese corrente para (tentar) fazer uma escolha "não puramente aleatória e uniforme" da nova batelada de exemplos. Em outras palavras, $T_{\text {double }}$ é uma mera adaptação de $T_{\text {SingleBatch }}$ para a possibilidade de não haver tempo suficiente para treinar com o dataset inteiro, e esta adaptação é feita com o "doubling trick".

\section{7}

\section{A estratégia Wrong Probably First (WPF)}

A estratégia $T_{W P F}$ é a nossa primeira tentativa de selecionar exemplos de forma diferente da aleatória uniforme. Se baseia no princípio $^{10}$ de que exemplos em que a hipótese corrente comete maior erro são mais úteis para treinar um novo modelo.

$T_{W P F}$ deve ser entendida como uma extensão de $T_{\text {double }}$, com a única (enorme) diferença de que, a partir da segunda iteração, os exemplos a serem adicionados não são sorteados uniformemente. Seus parâmetros são os seguintes:

$-\operatorname{frac}_{\text {start }} \in(0,1]$

- sample $_{\text {factor }} \in[1,+\infty)$

$-w_{\text {error }} \in[0,1]$

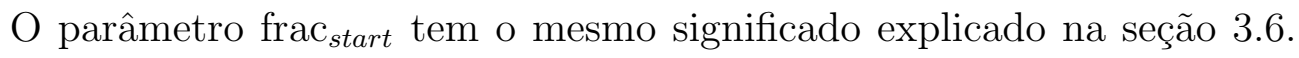
O parâmetro sample $_{\text {factor }}$ controla que fração dos exemplos livres (ainda não usados em treino) será avaliada para seleção dos novos exemplos (a cada iteração a partir da segunda). Já $w_{\text {error }}$ controla a importância do erro (cometido pela hipótese corrente) de um exemplo para determinar o peso deste exemplo no sorteio de novos exemplos. O significado preciso destes parâmetros ficará mais claro, adiante, quando explicaremos toda a mecânica de $T_{W P F}$.

Na primeira iteração, $T_{W P F}$ seleciona $m \cdot$ fracs $_{\text {start }}$ exemplos de forma aleatória uniforme ${ }^{11}$. Ainda de forma semelhante a $T_{\text {double }}$, a cada próxima iteração $i+1, T_{W P F}$ seleciona mais $\left|S_{i}\right|$ novos exemplos, onde $S_{i}$ é o conjunto de exemplos treinados na iteração $i$. Esta seleção, diferente do que ocorre em

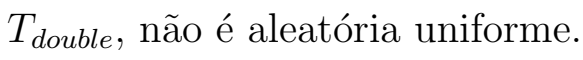

O sorteio (da batelada de exemplos a partir da segunda iteração) é feito em duas etapas. Primeiro, são sorteados uniformemente $\left|S_{i}\right| \cdot$ sample $_{\text {factor }}$ exemplos, que formam a amostra $A$. A cada iteração que não a primeira, a amostra $A$ muda.

\footnotetext{
${ }^{10}$ Isto não foi demonstrado e será verificado a partir de experimentos

${ }^{11}$ Exatamente como feito por $T_{\text {double }}$
} 
Em seguida, para cada exemplo $j$ da amostra $A$, calcula-se o erro de previsão $e_{j}$. Note que o $j$-ésimo exemplo de $A$ não é o $j$-ésimo exemplo do dataset. Cada exemplo é associado a um peso $w_{j}$, com

$$
w_{j}=w_{\text {error }} \cdot e_{j}^{\prime}+\left(1-w_{\text {error }}\right) \cdot \frac{1}{|A|}
$$

Aqui, $e_{j}^{\prime}$ é o erro de previsão normalizado, com $e_{j}^{\prime}=e_{j} / \sum_{k=1}^{|A|} e_{k}$. Essa transformação garante $\sum_{j=1}^{|A|} e_{j}^{\prime}=1$, que por sua vez garante $\sum_{j=1}^{|A|} w_{j}=1$

Finalmente, da amostra $A$ são sorteados $\left|S_{i}\right|$ exemplos, de acordo com os $\operatorname{pesos} w_{j}$.

Simplificadamente, cada exemplo da amostra $A$ é associada um peso que é função de uma parcela uniforme $(1 /|A|)$ e de uma parcela relacionada ao erro de previsão. A influência de cada parcela é controlada pelo parâmetro $w_{\text {error }}$. 


$\overline{\text { Algorithm } 4 T_{W P F}}$

function START: same as in $T_{\text {SingleBatch }}$

function CHOOSE_H: same as in $T_{\text {SingleBatch }}$

function SELECT_FIRST_EXAMPLES: same as in $T_{\text {double }}$

global variables: size $_{\text {next }_{b a t c h}}$, frac $_{\text {start }}, m=|X|, \mathbf{v}_{\text {free }_{\text {ids }}}$

more global variables: sample $_{\text {factor }}, w_{\text {error }}, \mathbf{v}_{\text {eval }}$ ids $_{\text {. }}$

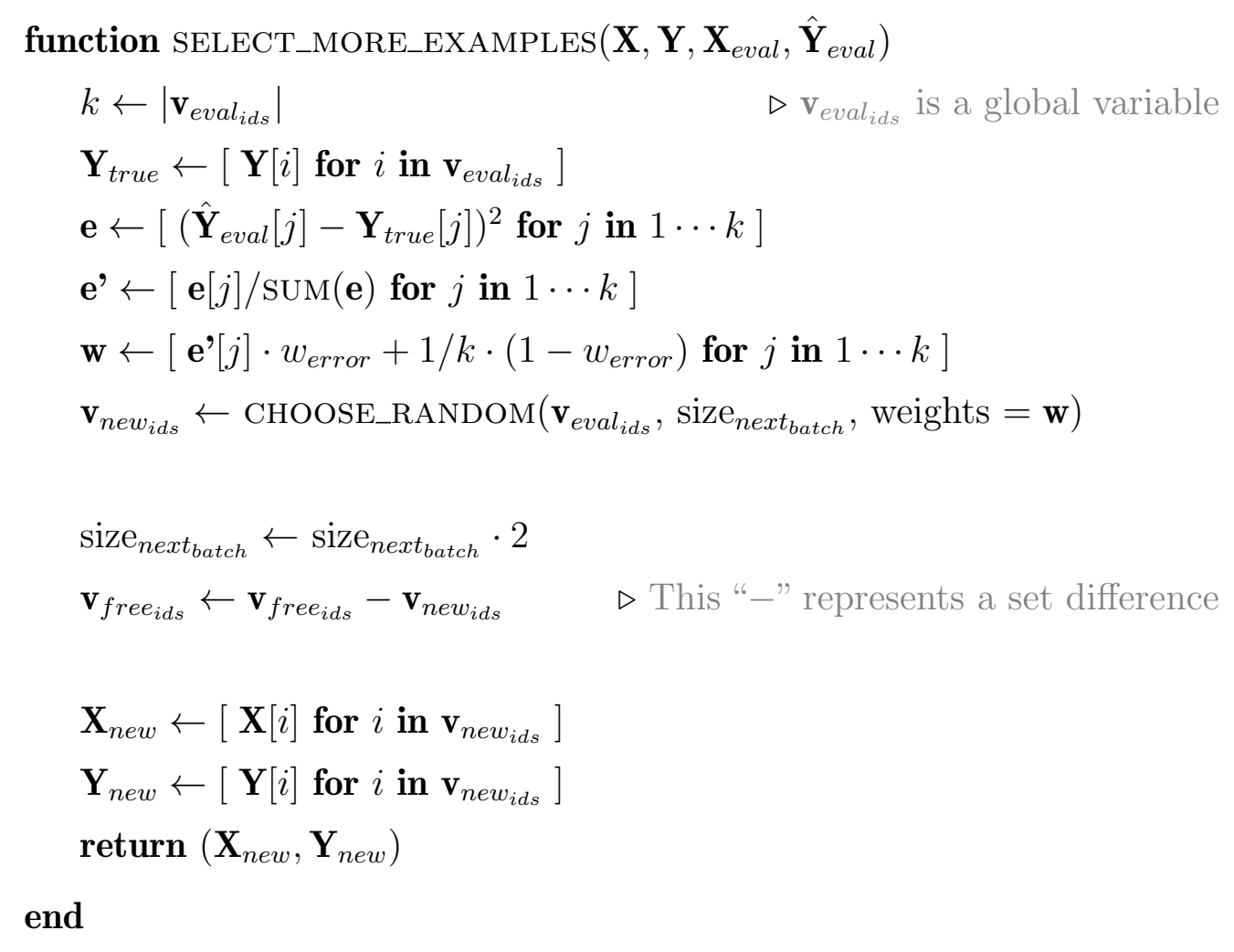

\section{function KEEP_EVALUATING}

if (já avaliou hipótese corrente) then

return $F A L S E$

else

return $T R U E$

end if

end

function SELECT_EVALUATION_EXAMPLes $(\mathbf{X}, \mathbf{Y})$

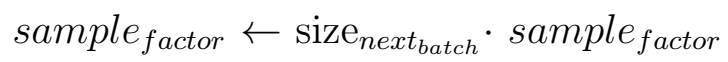

$\mathbf{v}_{\text {eval }_{\text {ids }}} \leftarrow$ CHOOSE_RANDOM $\left(\mathbf{v}_{\text {free }_{i d s}}\right.$, sample $\left._{\text {factor }}\right)$

return $\left[\mathbf{X}[i]\right.$ for $i$ in $\left.\mathbf{v}_{\text {eval }_{i d s}}\right]$ 
Assim como nas demais ocasiões, esse pseudocódigo não trata de casos de fronteira (degenerados). Em particular, se $\operatorname{SUM}(\mathbf{e})=0$, teríamos uma divisão por ZERO na execução da função SELECT_MORE_EXAMPLES. A propósito, $\operatorname{SUM}(\mathbf{e})$ denota a soma dos elementos do vetor e.

A chamada de função

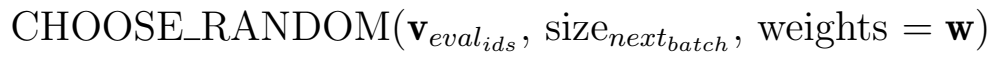

é diferente das chamadas feitas anteriormente, em $T_{\text {double }}$. Aqui, são sorteados size $_{\text {next }}$ batch $_{\text {elementos de }} \mathbf{v}_{\text {eval }}$ ids , sem reposição, mas este sorteio não é uniforme: os pesos de cada elemento são dados pelo vetor $\mathbf{w}$. Um detalhe de implementação: na verdade, sorteamos os índices - ou ponteiros - dos exemplos em $(\mathbf{X}, \mathbf{Y})$.

Também é importante reforçar outra diferença em relação à $T_{\text {double }}$ : esta etapa do sorteio não é feita a partir de todos os exemplos livres (ainda não usados em treino), mas sim dos exemplos para os quais a hipótese corrente foi avaliada. Isto porque este é um segundo sorteio - o primeiro deles foi feito na seleção de exemplos para teste, em SELECT_EVALUATION_EXAMPLES(X, Y).

A instrução

$$
\left(\hat{\mathbf{Y}}_{\text {eval }}[j]-\mathbf{Y}_{\text {true }}[j]\right)^{2}
$$

assume que a função de perda é o erro quadrático médio, de modo que a contribuição de cada exemplo é seu erro quadrático. Para adotar outra função de perda - como a diferença em módulo -, bastaria adaptar essa linha de código.

Por último, é instrutivo enxergar o comportamento de $T_{W P F}$ quando estressamos os parâmetros $w_{\text {error }}$ e sample sactor $_{\text {. }}$.

Note que se $w_{\text {error }}=0$, a seleção de exemplos ignora os erros e $T_{W P F}$ se torna $T_{\text {double }}$. O parâmetro $w_{\text {error }}$ pode ser visto como uma forma de priorizar a escolha de exemplos (i) por uma distribuição discreta uniforme ou (ii) por uma distribuição que dá mais peso aos exemplos com maior erro de previsão.

Finalmente, se sample $_{\text {factor }}=+\infty$, não haverá primeira etapa do sorteio a cada iteração a partir da segunda iteração, $T_{W P F}$ irá avaliar (rotular e calcular o erro) a hipótese corrente em todo o dataset ainda não treinado. O parâmetro sample $_{\text {factor }}$ pode ser visto como uma medida de quanto iremos explorar o dataset (espaço de busca) antes de inferir que exemplos serão escolhidos para treino.

A seção 3.11 contém um exemplo ilustrativo do funcionamento de $T_{W P F}$. 


\section{8}

\section{A estratégia Wrong Probably First + Best Hypothesis $(\mathrm{W}+\mathrm{BH})$}

Esta estratégia é quase idêntica à $T_{W P F}$. A única diferença é que, a cada iteração, $T_{W+B H}$ mantém como hipótese a ser retornada (valor guardado pela variável $\left.h_{\text {selected }}\right)$ a hipótese que teve o melhor resultado nas avaliações.

A cada iteração, $T_{W+B H}$ associa ao modelo que acabou de ser treinado um erro de avaliação, calculado com base nos erros $e_{j}$ da amostra $A$ (detalhada na seção 3.7). O valor de $h_{\text {selected }}$ só é atualizado quando o erro de avaliação do modelo corrente é menor do que o erro de avaliação do modelo apontado por $h_{\text {selected }}$.

É importante notar que $T_{W+B H}$ não faz nenhum processamento a mais do que $T_{W P F}$ : ao solicitar que $L$ rotule os exemplos da amostra $A$, também ganhamos uma estimativa do erro do modelo corrente na amostra $A$.

Finalmente, para tomar a decisão sobre qual hipótese manter apontada pela variável $h_{\text {selected }}$, algumas poucas adaptações devem ser feitas na inicialização da estratégia. Além disso, assumimos a existência de mais duas variáveis globals, error $_{h_{\text {selected }}}$ e error $_{h_{\text {curr }}}$, para manter intacatas as assinaturas das funções definidas em 3.1.

$\overline{\text { Algorithm } 5 T_{W+B H}}$

function START: same as in $T_{\text {SingleBatch }}$

function SELECT_FIRST_EXAMPLES: same as in $T_{\text {double }}$

function SELECT_MORE_EXAMPLES: same as in $T_{W P F}$

function KEEP_EVALUATION: same as in $T_{W P F}$

function SELECT_EVALUATION_EXAMPLES: same as in $T_{W P F}$

global variables: size $_{\text {next }}{ }_{b a t c h}$, frac $_{\text {start }}, m=|X|, \mathbf{v}_{\text {free }_{i d s}}$

more global variables: sample $e_{\text {factor }}, w_{\text {error }}, \mathbf{v}_{\text {eval }}$ ids

even more global variables: $\operatorname{error}_{h_{\text {curr }}}$, error $_{h_{\text {selected }}}$

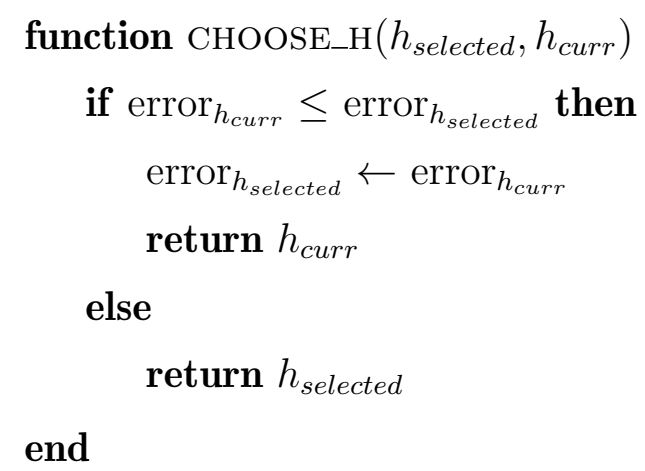




\section{9}

\section{A estratégia Uncertain Probably First (UPF)}

Uma outra maneira de não escolher exemplos de treino de forma puramente aleatória uniforme é associar a cada exemplo livre (exemplo que ainda não faz parte do conjunto de treino) uma incerteza sobre seu rótulo. Esta incerteza será usada por $T_{U P F}$ da mesma forma que o erro de previsão é utilizado por $T_{W P F}$ : no sorteio de novos exemplos de treino, quanto maior a incerteza sobre o rótulo de um exemplo, maior a chance de este exemplo ser sorteado.

$T_{U P F}$ deve ser visto como uma variação de $T_{W P F}$, com a única diferença sendo na maneira como os pesos são atribuídos aos exemplos da subamostra de teste (identificados pelos índices de $\mathbf{v}_{\text {eval }}$ ids $n$ pseudocódigo de $T_{W P F}$ ), dos quais alguns exemplos serão sorteados para compor o novo conjunto de treino.

Ao contrário da noção de erro de previsão, que sempre existe quando tratamos de aprendizado supervisionado, a noção de incerteza de previsão não é dada (não é necessária) pelo próprio problema que queremos resolver. Segue disso que a noção de incerteza depende da espécie de $L$. Se $L$ é uma Árvore de Regressão, por exemplo, podemos medir a incerteza de previsão de um exemplo $e$ pelo desvio-padrão dos rótulos que compõe o nó-folha a que $e$ foi direcionado (quando percorre esta árvore a partir da raiz). Já se $L$ for um modelo de ensemble ${ }^{12}$, a incerteza poderia ser associada a alguma medida de dispersão dos rótulos dados pelos modelos ao exemplo $e$.

Como, por premissa, $L$ deve ser visto como uma caixa-preta, faremos uma concessão à especificação de $T_{U P F}$ : na função de inicialização da estratégia, iremos conferir se $L$ é um dos algoritmos para os quais sabemos calcular incerteza de previsão ${ }^{13}$. Caso positivo, a estratégia segue seu curso; caso negativo, a estratégia aborta sua execução.

Nos experimentos que realizamos, o único $L$ para o qual estimamos uma incerteza foi $L=$ Random Forest. Para esse caso, a incerteza sobre um exemplo $e$ é o desvio-padrão dos rótulos de $e$ de acordo com cada árvore da Random Forest. Em outras palavras, para estimar a incerteza de $e$ (de acordo com uma Random Forest treinada, especificada), rotulamos $e$ de acordo com cada árvore do modelo, formando uma lista de valores (previsões do modelo), e em seguida calculamos o desvio-padrão dessa lista de valores.

Não apresentaremos o pseudocódigo de $T_{U P F}$, dada sua semelhança com $T_{W P F}$.

Os resultados experimentais de $T_{U P F}$ serão exibidos apenas na seção 4.9 .

\footnotetext{
${ }^{12} \mathrm{Um}$ modelo que combina modelos de aprendizado

${ }^{13}$ Isto é uma concessão porque a definição do problema não assume que temos essa informação sobre $L$
} 


\subsection{1}

\section{O paradigma de Active Learning}

A estratégia $T_{U P F}$ é inspirada no paradigma de Active Learning, em que exemplos não-rotulados são abundantes, mas obter os rótulos é custoso (é difícil, é caro ou exige tempo, conforme [16]). Isto porque, ao estimar a incerteza da previsão de exemplos, não usamos a informação do rótulo destes exemplos (apesar de disponível). É como se, ao querermos buscar $n$ exemplos para incrementar o conjunto de treino, tivéssemos uma subamostra com sample factor $\cdot n$ exemplos não-rotulados disponíveis. Diante dessa carência artificial de rótulos, a estratégia se baseia na incerteza de previsão dos exemplos dessa subamostra para escolher quais $n$ exemplos iremos rotular e consequentemente disponibilizar para $L$ treinar.

\subsection{0}

\section{Breve discussão sobre a complexidade de algumas etapas das estratégias}

As estratégias $T_{W P F}$ e $T_{W+B H}$ investem parte de seu tempo de treinamento em selecionar exemplos de forma aleatória não-uniforme.

Apesar de este trabalho ser essencialmente empírico, é válido observar que estas estratégias não pioram a complexidade do tempo total gasto em função do número exemplos treinados (em relação à estratégia $T_{\text {double }}$ ).

Isto porque, para treinar um conjunto de tamanho $n^{14}$, além das chamadas para treino do modelo idênticas às chamadas feitas por $T_{\text {double }}$, as estratégias $T_{W P F}$ e $T_{W+B H}$ executam as seguintes tarefas:

1. Sortear, de forma aleatória uniforme, $\mathcal{O}(n) \cdot$ sample $_{\text {factor }}$ exemplos

2. Rotular $\mathcal{O}(n) \cdot$ sample $_{\text {factor }}$ exemplos

3. Calcular o erro de previsão de $\mathcal{O}(n) \cdot$ sample $_{\text {factor }}$ exemplos

4. Sortear, de acordo com pesos, $\mathcal{O}(n)$ exemplos

Supondo que as tarefas de sortear, rotular e calcular erro de previsão não são mais caras do que treinar, o tempo gasto treinando é assintoticamente pelo menos tão grande quanto o tempo gasto com todas essas tarefas.

Na prática, mesmo que as estratégias não sejam assintoticamente mais caras, esse tempo gasto sem treinar pode resultar num subconjunto final de treino menor (do que o de $T_{\text {double }}$ ).

\footnotetext{
${ }^{14}$ Para treinar um conjunto de tamanho $n, T_{\text {double }}$ irá treinar conjuntos de tamanho $1,2,4, \cdots, n$. A soma de todos esses tamanhos (progressão geométrica de razão 2 e $\log (n)+1$ termos) é menor do que $2 n$.
} 
Supondo que, entre as tarefas sortear, rotular, calcular erro de previsão, a mais cara seja rotular, o tempo gasto sem treinar será menos impactante quando a complexidade de rotular for bem menor do que a de treinar. Este é o caso de muitos algoritmos de aprendizado ${ }^{15}$.

\subsection{1}

\section{Exemplo da estratégia Wrong Probably First (WPF)}

Apresentamos um exemplo ilustrativo da estratégia $T_{W P F}$ para um dataset com três grafias diferentes das letras A, B, C, D e E, totalizando 15 exemplos, cujo objetivo é identificar a posição da letra no alfabeto $(A=1, B=2, \ldots, E=$ 5). O erro é uma função das posições correta e prevista de cada exemplo. Assumimos os seguintes valores para os parâmetros de $T_{W P F}$ :

- $\operatorname{frac}_{\text {start }}=1 / 15$, de forma que o conjunto de treino inicial tem tamanho 1

- sample $_{\text {factor }}=2.5$

- $w_{\text {error }}=1 / 3$

Alguns esclarecimentos são essenciais para entender a figura:

1. Cada linha da figura 3.1 corresponde a uma iteração da estratégia.

2. Cada coluna corresponde a uma etapa de uma iteração

(a) A primeira etapa representa o conjunto de exemplos livres no início da iteração

(b) A segunda etapa representa o conjunto de exemplos escolhidos para serem avaliados (dos quais um subconjunto será escolhido para incrementar o conjunto de treino)

(c) A terceira etapa representa o conjunto de treino ao fim da iteração.

3. As letras em vermelho representam os exemplos escolhidos para a próxima etapa (da esquerda para a direita)

4. Os erros de previsão apresentados na coluna do meio são valores arbitrários. Não é possível deduzi-los (calcula-los) a partir da figura.

\footnotetext{
${ }^{15}$ Mas certamente não é o caso de todos. Atualizar os pesos de uma rede neural artificial (de acordo com um exemplo) é tão caro quanto calcular o rótulo deste exemplo.
} 


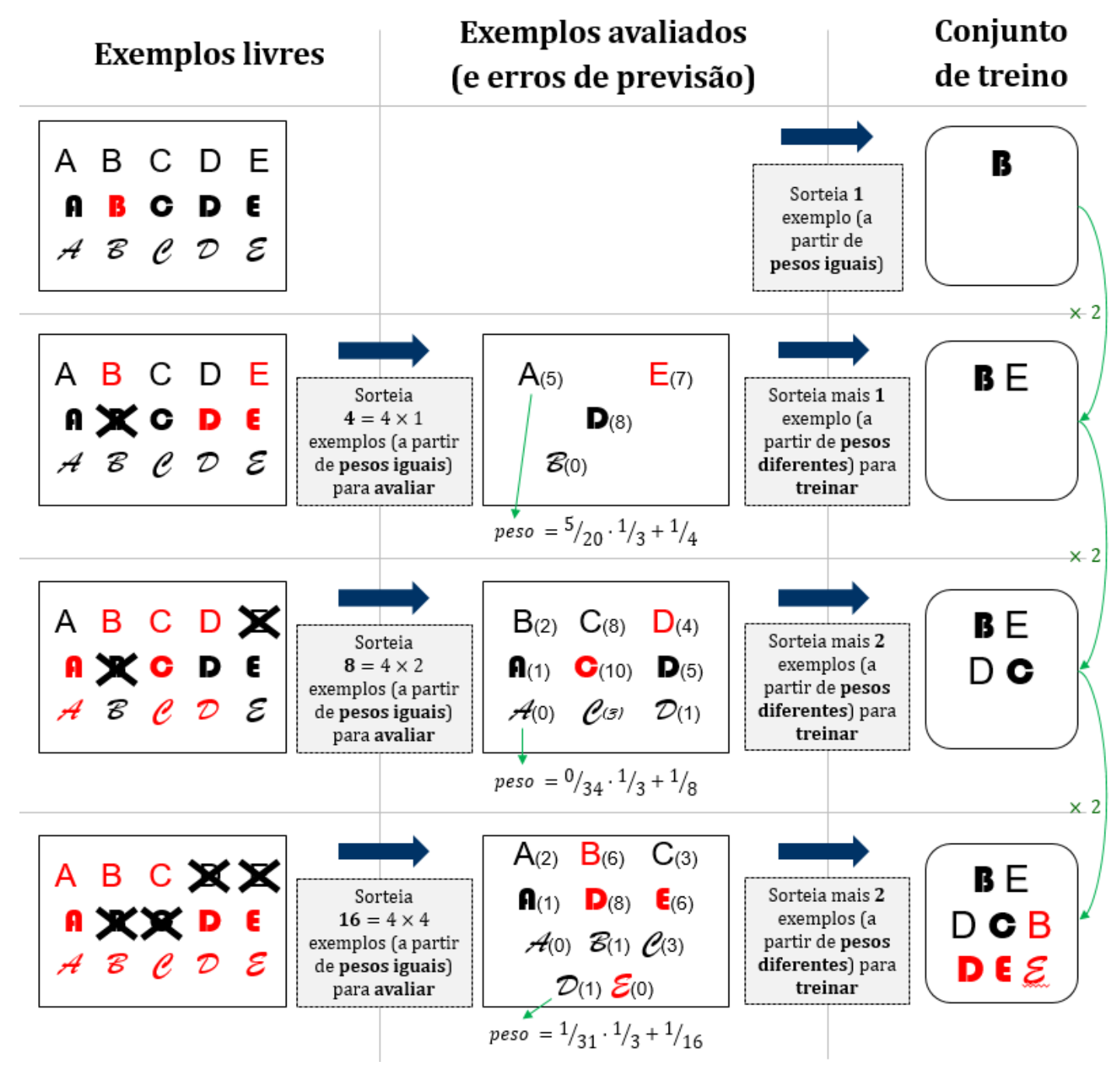

Figura 3.1: Exemplo com quatro iterações da estratégia $T_{W P F}$.

sample $_{\text {factor }}=4 \quad$ frac $_{\text {start }}=1 / 15 \quad w_{\text {error }}=1 / 3$ 


\section{4}

\section{Avaliação Experimental}

Este capítulo trata dos experimentos realizados para medir a qualidade das estratégias descritas na seção 3. Como premissa, assumimos que se houvesse tempo suficiente o melhor que poderíamos fazer é treinar $L$ com o dataset inteiro. Considerando a possibilidade de não haver tempo hábil, uma solução natural é selecionar exemplos aleatoriamente e ir aumentando gradualmente a parcela de exemplos treinados. Esta solução é a estratégia $T_{\text {double }}$.

Os experimentos, em síntese, consistem em verificar, ao longo do intervalo de tempo $\left(0, t_{\text {lim }}\right]^{1}$, a qualidade dos modelos produzidos pelas estratégias de seleção de exemplos (ou de crescimento gradual do dataset treinado).

O objetivo principal dos experimentos foi comparar as estratégias $T_{W+B H}$ e $T_{\text {double }}$.

\section{1}

\section{Datasets}

Foram realizados experimentos com 12 datasets, descritos na tabela 4.1. Os datasets foram retirados de dois repositórios: UCI e Kaggle. Cada dataset passou pelas seguintes transformações:

1. dados categóricos sem noção de ordem foram transformados em colunas com one hot encoding

2. dados categóricos com noção de ordem foram transformados em números inteiros a partir de 0

3. cada atributo foi normalizado ${ }^{2}$

${ }^{1} \mathrm{O}$ valor de $t_{\text {lim }}$ depende do par $(L$, dataset). Isso vai ser melhor explicado ao longo deste capítulo.

${ }^{2}$ A normalização foi feita com base nos dados do conjunto de treino e aplicada sobre o conjunto de treino e sobre o conjunto de teste 
Tabela 4.1: Datasets

\begin{tabular}{lll}
\hline Nome & \# exemplos & \# atributos \\
\hline superconductivty & 21.263 & 81 \\
hydraulic system valve condiciton & 2.205 & 154 \\
pmsm temperature & 998.070 & 8 \\
zap imoveis sp & 50.898 & 14 \\
dielectron & 99.915 & 16 \\
Bike-Sharing-Dataset hour & 17.379 & 31 \\
sgemm product & 24.1600 & 14 \\
default of credit card clients & 30.000 & 32 \\
SeoulBikeData & 8.760 & 15 \\
transcoding mesurment & 68.784 & 17 \\
spotify & 174.389 & 13 \\
tabular playground & 300.000 & 14 \\
\hline
\end{tabular}

Cada dataset foi embaralhado e em seguida separado em dois conjuntos: um conjunto de treino com $70 \%$ dos exemplos e um conjunto de teste com $30 \%$ dos exemplos.

\section{2}

\section{Algoritmos de aprendizado}

Foram realizados experimentos com 3 algoritmos de aprendizado:

- Support Vector Regression (SVR)

- Decision Tree Regressor

- Random Forest Regressor

Foram escolhidos algoritmos de aprendizado comumente usados em problemas de regressão. Não fizemos experimentos com Regressão Linear devido ao tempo de execução muito curto desse algoritmo (para treinar e rotular), o que torna difícil simular o cenário em que o tempo é um recurso escasso.

O algoritmo de Árvore de Regressão é o disponibilizado no pacote scikitlearn, de Python, e é baseado nos modelos descritos em [11] e [3]. Cada folha da árvore é associada a média de seus exemplos de treino. A configuração padrão do algoritmo neste pacote é tal que os nós da árvore são expandidos até o erro em cada nó folha ser zero (até que todos os exemplos do nó folha tenham o mesmo rótulo).

O algoritmo de Random Forest (Regressor) é um ensemble de Árvores de Regressão: o algoritmo treina 100 Árvores de Regressão em 100 subconjuntos 
de treino sorteados aleatoriamente. O rótulo de um exemplo corresponde à média dos rótulos de cada uma das 100 árvores.

O algoritmo de Support Vector Regression (SVR) também é o disponibilizado no pacote scikitlearn, de Python. Trata-se de uma implementação de Epsilon-Support Vector Regression, descrita em [18] e [4]. O algoritmo segue uma ideia bastante semelhante a de algoritmos de SVM de classificação. Dado uma tolerância $\epsilon>0$ e um dataset $\left\{\left(x_{1}, y_{1}\right), \ldots\left(x_{m}, y_{m}\right)\right\}$, procuramos a melhor curva da forma $f(x)=w \cdot x+b$ que satisfaz $\left|f\left(x_{i}\right)-y_{i}\right| \leq \epsilon, \quad i=1 \ldots m$. A melhor curva é aquela que minimiza $\|w\|^{2}$. Quando não existe função que aproxima todos os pontos $x_{i}$ a $y_{i}$ dentro da tolerância $\epsilon$ - que é o equivalente, para classificação, de não existir hiperplano que separa perfeitamente os pontos —, o problema de minimização é reescrito com variáveis de folga, para permitir (mas penalizar) que alguns pontos estejam a uma distância maior do que $\epsilon$ da curva. Em outras palavras, é um problema de minimização, em que queremos achar uma curva (hiperplano) que minimiza distâncias (entre a curva e os rótulos $y_{i}$ ), mas distâncias menores do que $\epsilon$ são toleradas (somam zero a função-objetivo a ser minimizada).

\section{3}

\section{Erro esperado e erro empírico}

Na teoria de aprendizado estatístico ([17]), o erro esperado de uma hipótese $h$ é dado por ${ }^{3}$

$$
L_{\mathcal{D}}(h) \stackrel{\text { def }}{=} \underset{(x, y) \sim \mathcal{D}}{\mathbb{E}}[\ell(h(x), y)]
$$

, em que:

- $\mathcal{D}$ é a distribuição de probabilidade dos exemplos ${ }^{4}$

- $\ell$ é a função de perda $\ell: \mathbb{R} \times \mathbb{R} \rightarrow \mathbb{R}$

Como por premissa não conhecemos $\mathcal{D}$, calcularemos o erro empírico de $h$ (sobre um dataset ou uma amostra $(X, Y)=S$ ), dado por

$$
L_{S}(h) \stackrel{\text { def }}{=} \frac{1}{|S|} \sum_{(x, y) \in S} \ell(h(x), y)
$$

Finalmente, como esta dissertação trata de problemas de regressão, utilizamos como função de perda $\ell$ o erro quadrático.

\footnotetext{
${ }^{3}$ A definição pode ser ainda mais geral, e esta é uma simplificação para o caso de aprendizado supervisionado em problemas de regressão

${ }^{4}$ No caso geral de aprendizado supervisionado, a distribuição de probabilidade é sobre o conjunto $\mathcal{Z}=\mathcal{X} \times \mathcal{Y}$
} 


$$
L_{S}(h) \stackrel{\text { def }}{=} \frac{1}{|S|} \sum_{(x, y) \in S}\left(h\left(x_{i}\right)-y_{i}\right)^{2} \stackrel{\text { def }}{=} \frac{1}{m} \sum_{i=1}^{m}\left(h\left(x_{i}\right)-y_{i}\right)^{2}
$$

O erro empírico é calculado sobre um conjunto de exemplos que não fez parte do treino, chamado conjunto de teste. Este conjunto não é enxergado pela estratégia.

\section{4}

\section{Medida de qualidade de uma estratégia}

A medida de qualidade de uma estratégia num instante de tempo $t$ sobre um input $\left(X, Y, L, t_{\text {lim }}\right)$ e um dataset de teste $\left(X_{\text {test }}, Y_{\text {test }}\right)$ - é o erro empírico da hipótese apontada por $h_{\text {selected }}$ no instante $t$, erro esse medido sobre $\left(X_{\text {test }}, Y_{\text {test }}\right)$. Lembramos aqui que $h_{\text {selected }}$ é, por definição, a hipótese que a estratégia selecionaria se o experimento terminasse naquele instante - e que isto muda ao longo do tempo, conforme a estratégia obtém novas hipóteses de $L$.

Como este trabalho é sobretudo empírico, apresentamos duas maneiras de medir a qualidade de uma estratégia: o erro do modelo retornado ao final do experimento e o erro do modelo ao longo do experimento. A partir daqui, usaremos como abuso de notação o termo "erro da estratégia" como sinônimo de "erro da hipótese apontada pela estratégia".

Dado um input $\left(X, Y, L, t_{\text {lim }}\right)$ e um dataset de teste $\left(X_{\text {test }}, Y_{\text {test }}\right)$, defina para a estratégia $T$ :

- $\operatorname{modelo}_{T}(t)=$ modelo (treinado) apontado por $T$ no instante $t$ (ou simplesmente o valor de $h_{\text {selected }}$ no instante $t$ )

- $\operatorname{erro}_{T}(t)=$ erro empírico de $\operatorname{modelo}_{T}(t)$ aplicado sobre $\left(X_{\text {test }}, Y_{\text {test }}\right)$

- erro $_{\text {full }}=$ erro empírico do modelo que treina com $(X, Y)$ sem restrição de tempo sobre $\left(X_{\text {test }}, Y_{\text {test }}\right)$ (ou simplesmente o erro empírico da hipótese retornada por $\left.T_{\text {SingleBatch }}\right)$

- $\operatorname{erro}_{\text {rel }}(t)=\operatorname{erro}_{T}(t) /$ errofull

A justificativa para a definição e o uso $\operatorname{erro}_{r e l}(t)$ é a seguinte: como estamos tratando de problemas de regressão, para facilitar a comparação entre erros de uma estratégia $T$ em dois datasets distintos, usamos como medida de performance o erro de $T$ em relação ao erro do modelo que treina com todo o dataset de treino $(X, Y)$. 
Cada trio (dataset, learner, teacher) foi rodado com 5 seeds, que influenciam a inicialização do learner. O valor de $\operatorname{erro}_{T}(t)$, portanto, é uma média do valor do erro para cada uma das seeds.

Finalmente, esclarecemos que colher estas estatísticas demanda tempo, mas este tempo não afetou o tempo-limite de execução de cada experimento. A cada instante $t$ em que erro $_{r e l}(t)$ é calculado, é como se parássemos o cronômetro que regula o tempo de execução da estratégia.

Em resumo, dado o input $\left(X, Y, L, t_{\text {lim }}\right)$ um dataset de teste $\left(X_{\text {test }}, Y_{\text {test }}\right)$, analisaremos a qualidade de uma estratégia $T$ de duas maneiras:

1. Pelo valor de $\mathrm{erro}_{r e l}\left(t_{l i m}\right)$, erro empírico ao final do experimento

2. Pelo gráfico de $\operatorname{erro}_{r e l}($.$) em função do tempo, que mostra o erro$ empírico ao longo do experimento

\section{5}

\section{Ambiente computacional dos experimentos}

Os experimentos foram realizados num processador Core i9-7900X 3.3GHz, com 128GB RAM DDR4, no Windows 10, na linguagem de programação Python. Os learners foram retirados do pacote scikitlearn ([15]) e utilizados com seus parâmetros pré-definidos ${ }^{5}$.

Para as estratégias $T_{W P F}$ e $T_{W+B H}$, a não ser que seja mencionado algo diferente, utilizamos os valores sample $_{\text {factor }}=4$ e $w_{\text {error }}=1 / 3$. Na seção 4.8 , mostramos que a performance dessas estratégias se mantém superior à de $T_{\text {double }}$ para diversos outros valores de sample $_{\text {factor }}$ e $w_{\text {error }}$.

Para todas as estratégias (exceto $T_{\text {SingleBatch }}$, que não tem parâmetros), foi utilizado frac start $=1 \%$.

\section{6}

\section{Resultados}

Os resultados comparam a performance das estratégias $T_{W+B H}$ e $T_{\text {double }}$ conforme o exposto na seção 4.4: pelo erro das estratégias ao fim do tempo-limite e pelo erro da estratégias ao longo do tempo.

O tempo-limite - para um par $(L$, dataset $)$ - foi estabelecido como o tempo para $L$ treinar com todo o conjunto de treino $(X, Y)$, sujeito ao limite

\footnotetext{
${ }^{5}$ Isso explica por que não estimamos a incerteza da previsão das Árvores de Regressão para a estratégia $T_{W P F}$ : por padrão, as árvores têm altura ilimitada e os nós são expandidos até que cada folha seja pura (todos os exemplos de uma folha têm o mesmo rótulo)
} 
superior de 5 minutos. Este teto de 5 minutos foi arbitrado para permitir análises de sensibilidade dos parâmetros de $T_{W+B H}$. No apêndice $\mathrm{A}$, exibimos evidências de que tempos diferentes não afetariam a direção dos resultados.

A tabela 4.6 exibe os erros dos modelos treinados por $T_{\text {double }}$ e $T_{W+B H}$ para cada par ( $L$, dataset) listado nas seções 4.2 e 4.1 .

\begin{tabular}{|c|c|c|c|c|c|c|}
\hline \multirow[b]{2}{*}{ Dataset } & \multicolumn{2}{|c|}{ Árv. de Regressão } & \multicolumn{2}{|c|}{ Random Forest } & \multicolumn{2}{|c|}{ SVR } \\
\hline & $T_{\text {double }}$ & $T_{W+B H}$ & $T_{\text {double }}$ & $T_{W+B H}$ & $T_{\text {double }}$ & $T_{W+B H}$ \\
\hline superconductivty & 1.57 & 1.51 & 1.35 & 1.18 & 1.04 & 0.99 \\
\hline hydraulic system & 1.46 & 1.63 & 1.21 & 1.28 & 1.04 & 0.97 \\
\hline pmsm temperature & 1.96 & 1.64 & 2.66 & 1.84 & 1.17 & 1.08 \\
\hline zap imoveis sp & 1.24 & 1.28 & 1.69 & 1.08 & 1.00 & 0.99 \\
\hline dielectron & 1.38 & 1.18 & 1.65 & 1.26 & 1.22 & 1.01 \\
\hline Bike-Sharing-hour & 1.29 & 1.25 & 1.26 & 1.09 & 1.03 & 1.00 \\
\hline sgemm product & 4.74 & 2.40 & 5.38 & 2.00 & 2.12 & 1.19 \\
\hline default of credit card & 0.99 & 0.97 & 1.01 & 1.04 & 1.00 & 0.99 \\
\hline SeoulBikeData & 1.12 & 1.13 & 1.16 & 1.06 & 1.04 & 1.00 \\
\hline transcoding mesurment & 0.98 & 1.02 & 1.00 & 1.05 & 1.00 & 0.98 \\
\hline spotify & 1.11 & 1.23 & 1.05 & 1.01 & 1.03 & 1.00 \\
\hline tabular playground & 1.01 & 1.02 & 1.01 & 1.01 & 1.03 & 1.02 \\
\hline Média das médias & 1.57 & 1.36 & 1.70 & 1.24 & 1.14 & 1.02 \\
\hline$\# \operatorname{erro}_{T_{W+B H}}<\operatorname{erro}_{T_{\text {Double }}}$ & & / 12 & & 12 & & / 12 \\
\hline
\end{tabular}

Tabela 4.2: Erros dos modelos finais, das estratégias $T_{\text {double }}$ e $T_{W+B H}$. Os valores com fundo cinza mostram que estratégia foi melhor em cada caso (cada par Learner, dataset)

O valor 1.16 (na linha SeoulBikeData, coluna Random Forest / $T_{\text {double }}$ ) se lê da seguinte forma: ao fim do experimento com o algoritmo de aprendizado $L=$ "Random Forest" e o dataset SeoulBikeData, a estratégia $T_{\text {double }}$ teve um erro empírico $16 \%$ superior ao erro empírico da hipótese que $L$ retorna ao treinar com todo o dataset.

Para cada um dos learners, $T_{W+B H}$ teve em média um erro final inferior ao erro final de $T_{\text {double }}$. No caso das Árvores de Regressão, no entanto, $T_{\text {double }}$ se saiu melhor em metade dos datasets. Para os outros dois learners, $T_{W+B H}$ foi 
melhor na maioria dos datasets. Em particular, para o SVR, $T_{W+B H}$ produziu um modelo final com erro de teste inferior ao erro do modelo produzido por $T_{\text {double }}$ em todos os datasets.

As figuras 4.1, 4.2 e 4.3 exibem o erro das estratégias ao longo do tempo, para cada um dos learners. Os resultados sugerem que as estratégias são competitivas para Árvores de Regressão e que $T_{W+B H}$ é superior $T_{\text {double }}$ nos demais casos - RandomForest e SVR. Neste contexto, a superioridade é evidenciada pelo gráfico de $T_{W+B H}$ estar abaixo (com erro menor) do gráfico de $T_{\text {double }}$. 

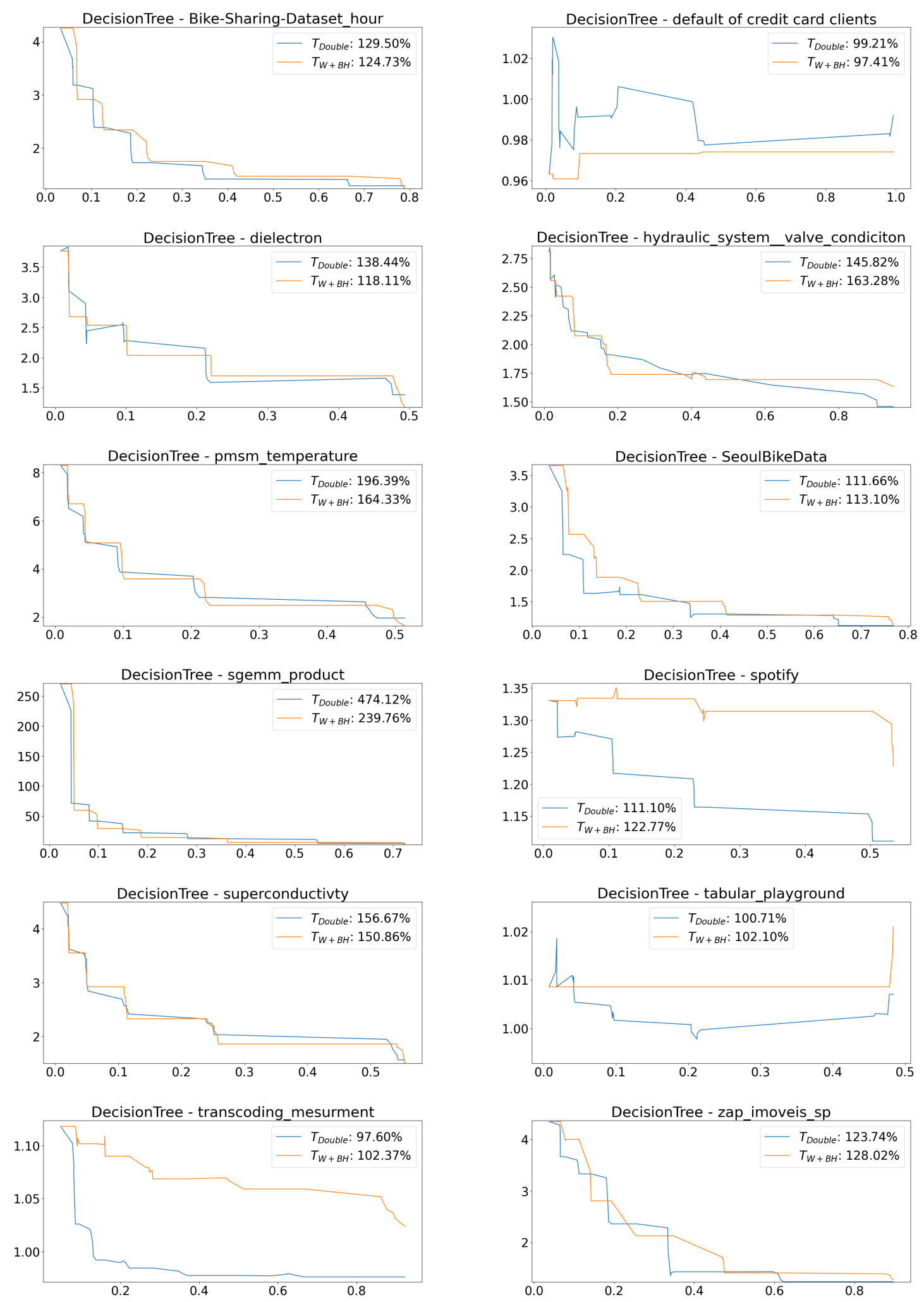

Figura 4.1: Erro médio (eixo vertical) ao longo do tempo (eixo horizontal) no conjunto de teste para as estratégias $T_{\text {double }}$ e $T_{W+B H}$, com $L=$ Árvores de Regressão. A legenda exibe o erro médio ao fim do experimento. 

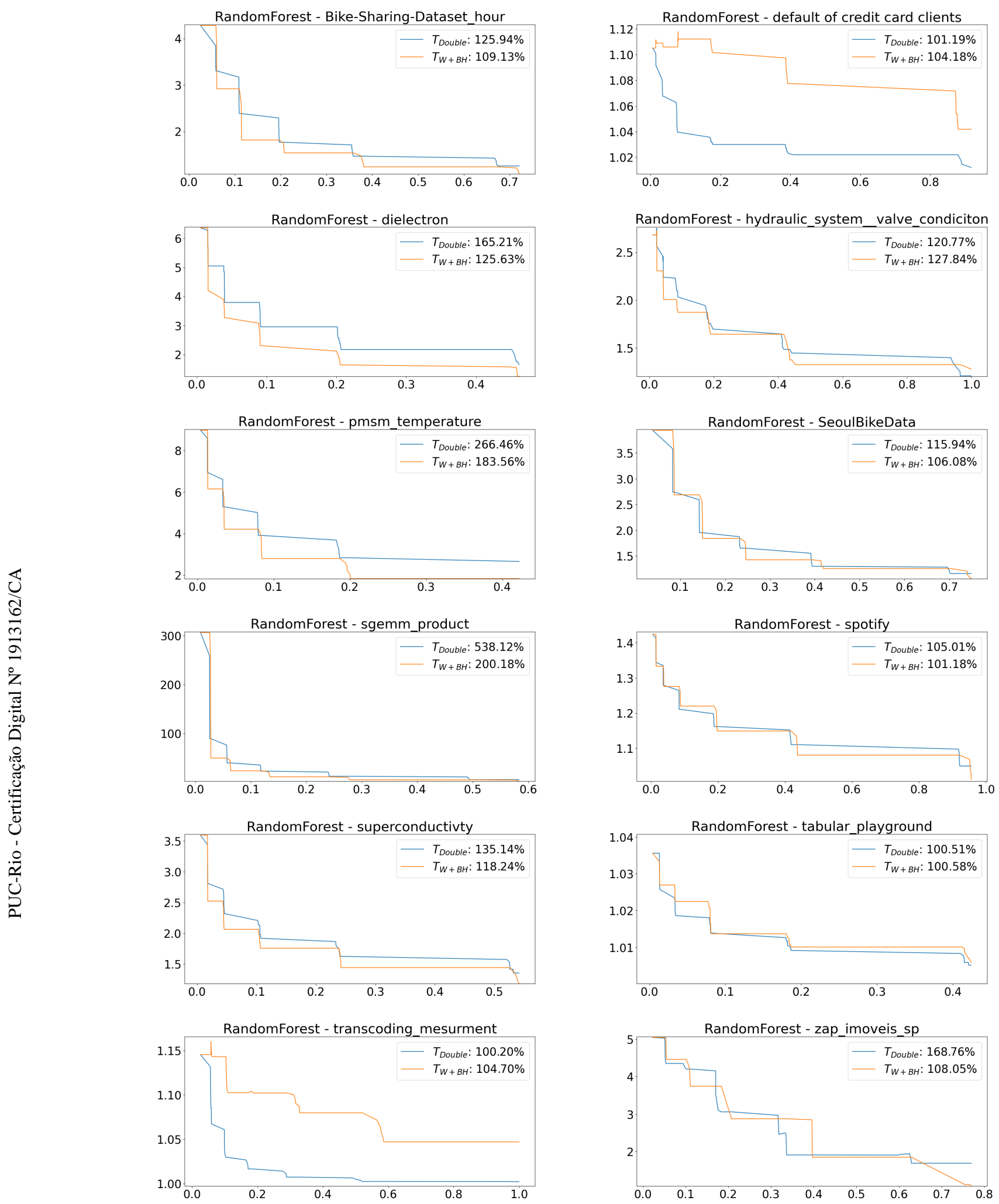

Figura 4.2: Erro médio (eixo vertical) ao longo do tempo (eixo horizontal) no conjunto de teste para as estratégias $T_{\text {double }}$ e $T_{W+B H}$, com $L=$ Random Forest. A legenda exibe o erro médio ao fim do experimento. 

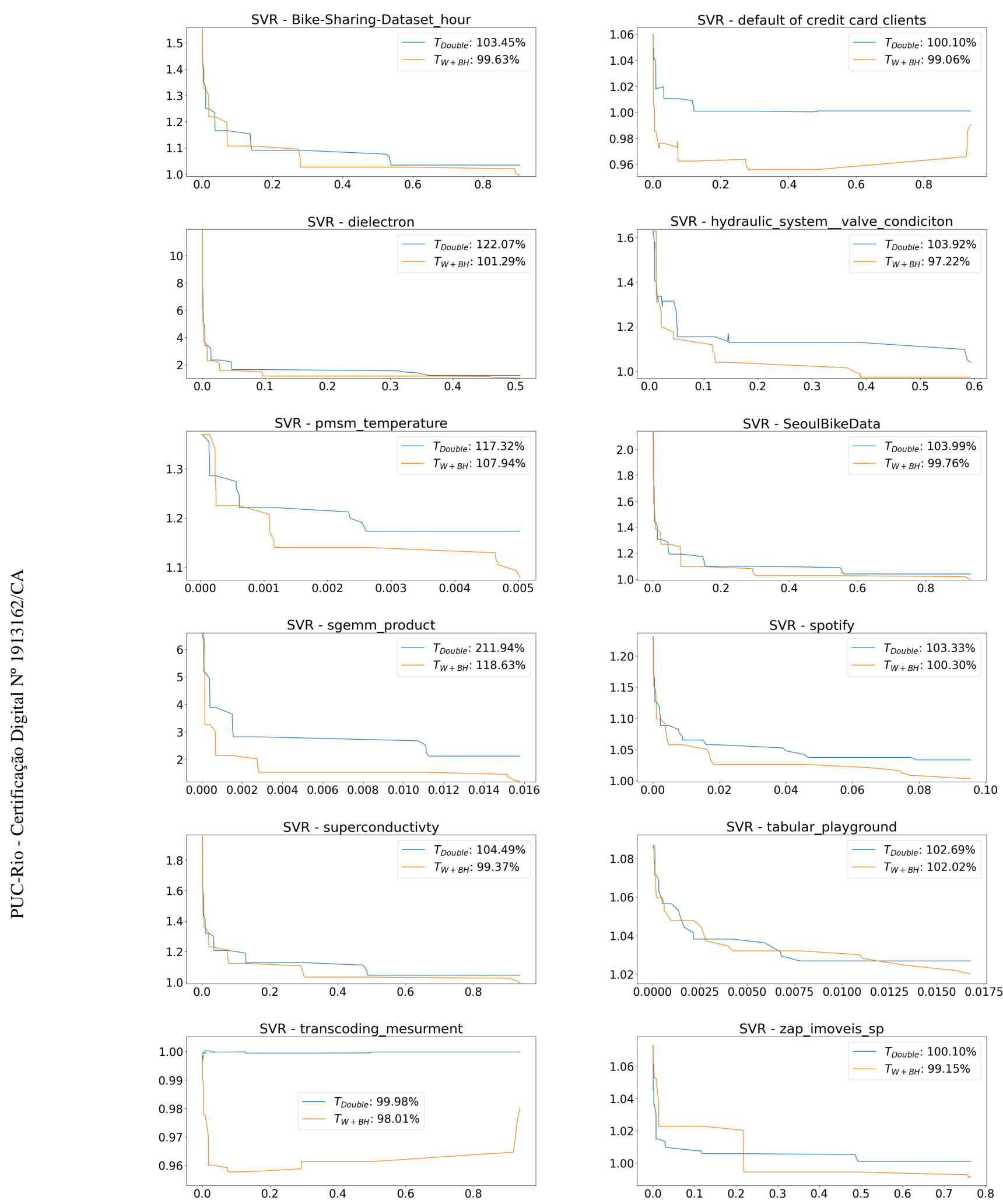

Figura 4.3: Erro médio (eixo vertical) ao longo do tempo (eixo horizontal) no conjunto de teste para as estratégias $T_{\text {double }}$ e $T_{W+B H}$, com $L=$ SVR. A legenda exibe o erro médio ao fim do experimento. 


\subsection{1}

\section{Alguns resultados para Wrong Probably First}

As estratégias $T_{W P F}$ e $T_{W+B H}$ são muito semelhantes. A única distinção — conforme exibido pelos pseudocódigos das seções 3.7 e 3.8 - é a política de troca de hipótese: enquanto $T_{W P F}$ sempre seleciona a última hipótese treinada por $L, T_{W+B H}$ só troca de hipótese quando a última hipótese treinada $\left(h_{c u r r}\right)$ teve um erro empírico (no conjunto de avaliação) melhor do que o da hipótese atualmente apontada $\left(h_{\text {selected }}\right)^{6}$.

Esta semelhança sugere que as estratégias terão desempenho semelhantes, o que é evidenciado pelos gráficos da figura 4.4, em que as linhas representando o erro de cada uma das estratégias praticamente coincidem.

Nestes experimentos, em vez de um gráfico por par ( $L$, dataset), mostramos um gráfico para cada $L$. Cada gráfico corresponde a uma média dos gráficos fixando $L$ e variando o dataset.

Concluímos que $T_{W+B H}$ raramente mantém uma hipótese que não a última treinada, mas, quando toma essa decisão, geralmente o resultado é positivo.

${ }^{6} \mathrm{Ou}$ quando nenhuma hipótese existe ainda, caso em que $h_{\text {selected }}=N U L L$ ! 

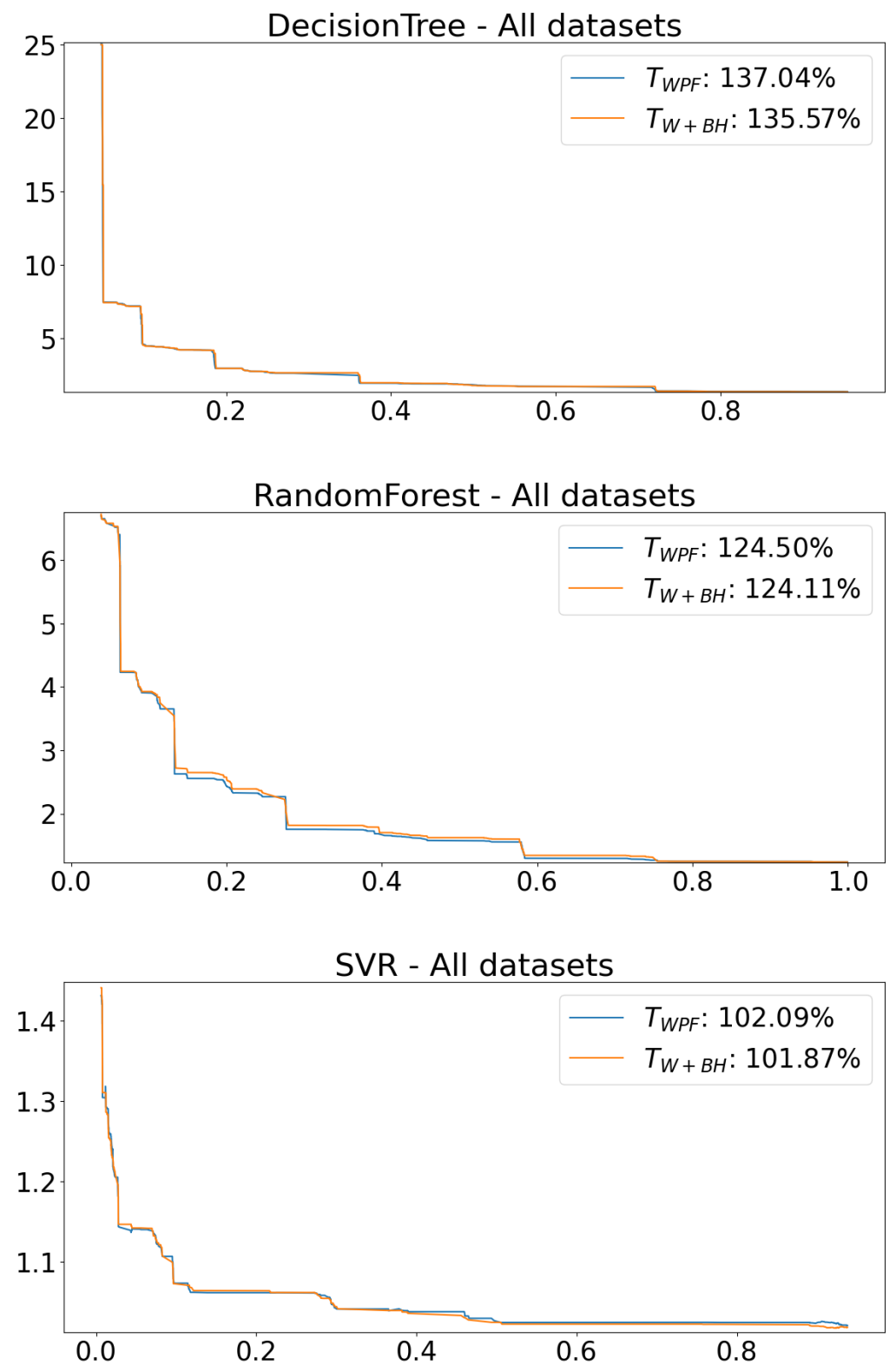

Figura 4.4: Erro médio (eixo vertical) ao longo do tempo (eixo horizontal) no conjunto de teste para as estratégias $T_{W+B H}$ e $T_{W P F}$. A legenda exibe o erro médio ao fim do experimento.

\section{7}

\section{O tamanho do conjunto de treino de cada estratégia}

A premissa de que exemplos em que a hipótese corrente comete maior erro são mais úteis para treinar um novo modelo é a motivação para as estratégias $T_{W P F}$ e $T_{W+B H}$. Mesmo que isso seja verdade, este tempo gasto avaliando exemplos reduz o tempo de treinamento, o que se traduz em conjuntos de treino finais menores (ou em hipóteses finais treinadas com menos exemplos do que 
as de $\left.T_{\text {double }}\right)$. A tabela 4.7 mostra a relação entre o tamanho do conjunto de treino final de $T_{W+B H}$ e de $T_{\text {double }}$ - nos dois casos em comparação ao tamanho total do dataset - , destacando em negrito os casos em que houve diferença (a favor de $T_{\text {double }}$, sempre).

Nem sempre os números da tabela serão aproximadamente uma potência de 2 , porque cada entrada da tabela é uma média de 5 experimentos ( 5 valores de seed).

\begin{tabular}{|c|c|c|c|c|c|c|}
\hline \multirow[b]{2}{*}{ Dataset } & \multicolumn{2}{|c|}{ Árv. de Regressão } & \multicolumn{2}{|c|}{ Random Forest } & \multicolumn{2}{|c|}{ SVR } \\
\hline & $T_{\text {double }}$ & $T_{W+B H}$ & $T_{\text {double }}$ & $T_{W+B H}$ & $T_{\text {double }}$ & $T_{W+B H}$ \\
\hline superconductivty & 0.32 & 0.32 & 0.32 & 0.32 & 0.64 & 0.64 \\
\hline hydraulic system & 0.50 & 0.37 & 0.62 & 0.37 & 0.62 & 0.28 \\
\hline pmsm temperature & 0.32 & 0.32 & 0.19 & 0.16 & 0.08 & 0.08 \\
\hline zap imoveis sp & 0.32 & 0.32 & 0.32 & 0.32 & 0.64 & 0.64 \\
\hline dielectron & 0.32 & 0.32 & 0.32 & 0.32 & 0.64 & 0.64 \\
\hline Bike-Sharing-hour & 0.32 & 0.32 & 0.32 & 0.32 & 0.64 & 0.64 \\
\hline sgemm product & 0.32 & 0.32 & 0.32 & 0.32 & 0.16 & 0.16 \\
\hline default of credit card & 0.51 & 0.07 & 0.64 & 0.64 & 0.64 & 0.64 \\
\hline SeoulBikeData & 0.32 & 0.32 & 0.32 & 0.32 & 0.64 & 0.64 \\
\hline transcoding mesurment & 0.32 & 0.32 & 0.45 & 0.32 & 0.64 & 0.64 \\
\hline spotify & 0.32 & 0.32 & 0.64 & 0.64 & 0.32 & 0.32 \\
\hline tabular playground & 0.32 & 0.13 & 0.32 & 0.32 & 0.16 & 0.16 \\
\hline
\end{tabular}

Tabela 4.3: Tamanho relativo dos modelos finais. Os valores com fundo cinza mostram que estratégia selecionou mais exemplos em cada caso (cada par Learner, dataset)

De fato, em alguns casos o tempo gasto por $T_{W+B H}$ escolhendo exemplos para treino (em vez de simplesmente sortear alguns exemplos) ocasiona que a estratégia rode uma iteração ${ }^{7}$ a menos.

Em 7 pares $(L$, dataset $)$ tivemos $T_{\text {double }}$ com conjunto de treino final maior do que o de $T_{W+B H}$. Desses 7 , em 4 deles $T_{\text {double }}$ teve desempenho superior.

\footnotetext{
${ }^{7}$ Vários cientistas da computação usam as palavras "iteragir" e "interagir" sem distinção, o que é um erro. Curiosamente, para falar de estratégias de seleção de exemplos, qualquer uma das duas palavras serve
} 

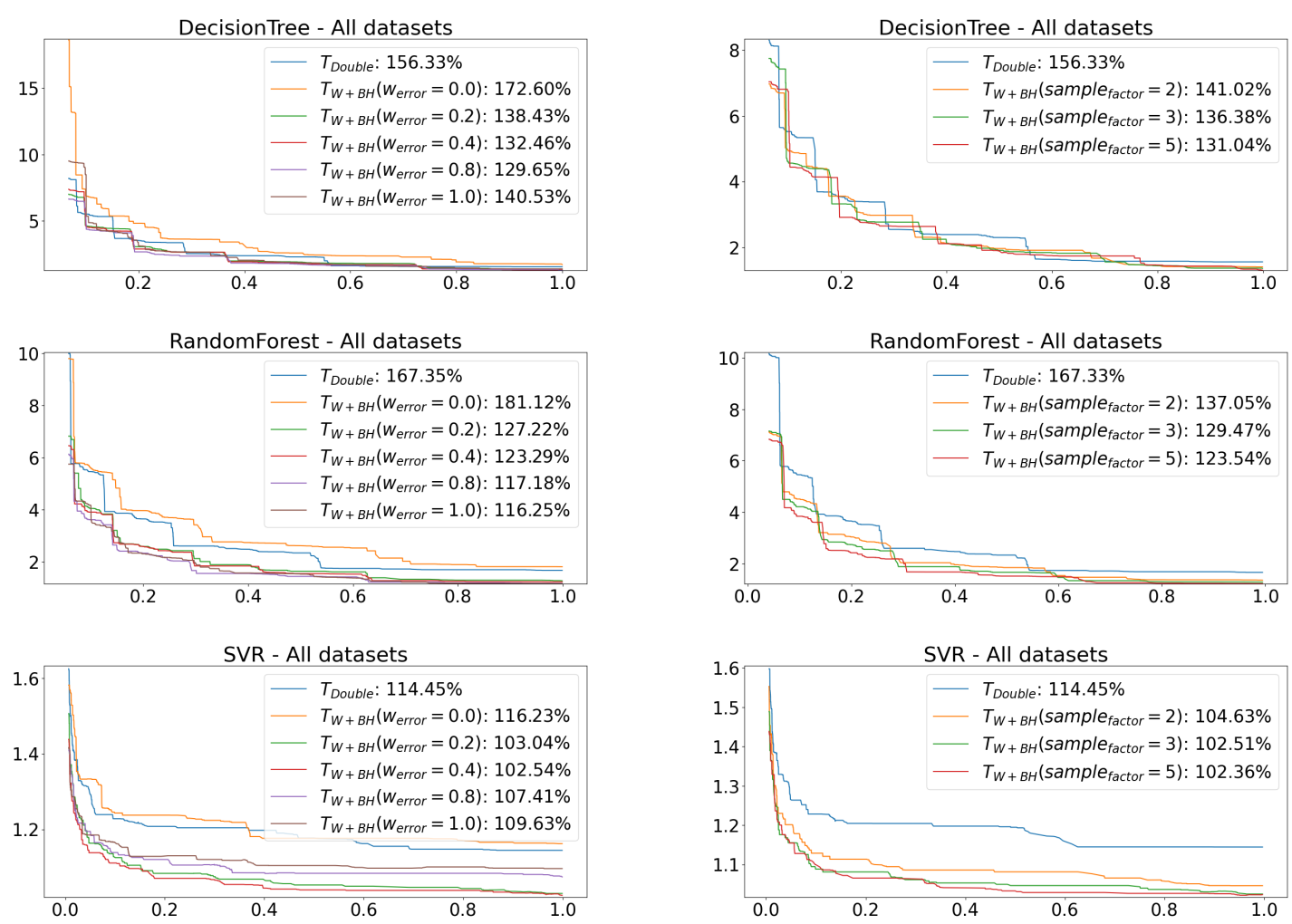

Figura 4.5: Erro médio (eixo vertical) ao longo do tempo (eixo horizontal) no conjunto de teste para a estratégia $T_{W+B H}$, variando os parâmetro $w_{\text {error }} \mathrm{e}$ sample $_{\text {factor }}$. A legenda exibe o erro médio ao fim do experimento.

Considerando que no geral $T_{\text {double }}$ ganhou de $T_{W+B H}$ em apenas 10 dos 36 pares, os resultados sugerem o que é esperado: que conjuntos de treino maiores todo o resto constante - geram modelos melhores (com menor erro empírico).

\section{8}

\section{Análise de sensibilidade}

A estratégia $T_{W+B H}$ tem dois parâmetros a mais do que a estratégia aleatória $T_{\text {double }}$ : sample fator e $w_{\text {error }}$. Conduzimos experimentos para demonstrar que, apesar de a escolha destes parâmetros influenciar a qualidade do modelos de aprendizado retornados pela estratégia, em geral a estratégia $T_{W+B H}$ vai ser superior a $T_{\text {double }}$ para um intervalo grande de parâmetros utilizados. A figura 4.5 exibe o erro empírico ao longo do tempo no conjunto de teste para estas duas estratégias.

Quando fazemos $w_{\text {error }}$ variar entre 0.2 e 0.8 , mantemos inalterado sample $_{\text {factor }}=4$, conforme os valores apresentados na seção 4.5. Analogamente, quando fazemos sample $_{\text {fator }}$ variar entre 2 e 5 , mantemos inalterado $w_{\text {error }}=$ $1 / 3$, conforme os valores apresentados na seção 4.5.

Nestes experimentos, em cada uma das duas análises de sensibilidade, em 
vez de um gráfico por par $(L$, dataset), mostramos um gráfico para cada $L$. Cada gráfico corresponde a uma média dos gráficos fixando $L$ e variando o dataset. A estratégia $T_{W+B H}\left(w_{\text {error }}=0.0\right)$ é um caso degenerado da estratégia $T_{W+B H}-$ corresponde ao $T_{\text {Double }}$, com a desvantagem do tempo gasto rotulando exemplos de forma desnecessária, uma vez que estes rótulos (ou seus erros) não serão usados no sorteio de exemplos.

\section{9}

\section{Experimentos com a estratégia baseada em incerteza}

A estratégia $T_{U P F}$ sorteia novos exemplos de treino com base na incerteza de previsão destes exemplos. Seus detalhes foram descritos na seção 3.9.

Só fizemos experimentos com $L=$ Random Forest. Para Árvores de Regressão, as configurações pré-estabelecidas pelo pacote scikitlearn geram uma árvore com erro zero sobre o conjunto de treino (cada nó é expandido até que não haver exemplos com rótulos diferentes).

Os gráficos da figura 4.6 e a tabela 4.9 comparam a qualidade das estratégias $T_{\text {double }}$ e $T_{U P F}$. A estratégia $T_{U P F}$ teve desempenho inferior à $T_{\text {double }}$ na maioria dos casos (datasets).

No entanto, se nos restringirmos aos casos em que $T_{U P F}$ conseguiu treinar com um conjunto tão grande quando o de $T_{\text {double, a estratégia se mostra }}$ competitiva. Isto sugere que a explicação para o desempenho ruim de $T_{U P F}$ foi o tempo gasto inferindo que exemplos usar para treino (em vez de treinando). 

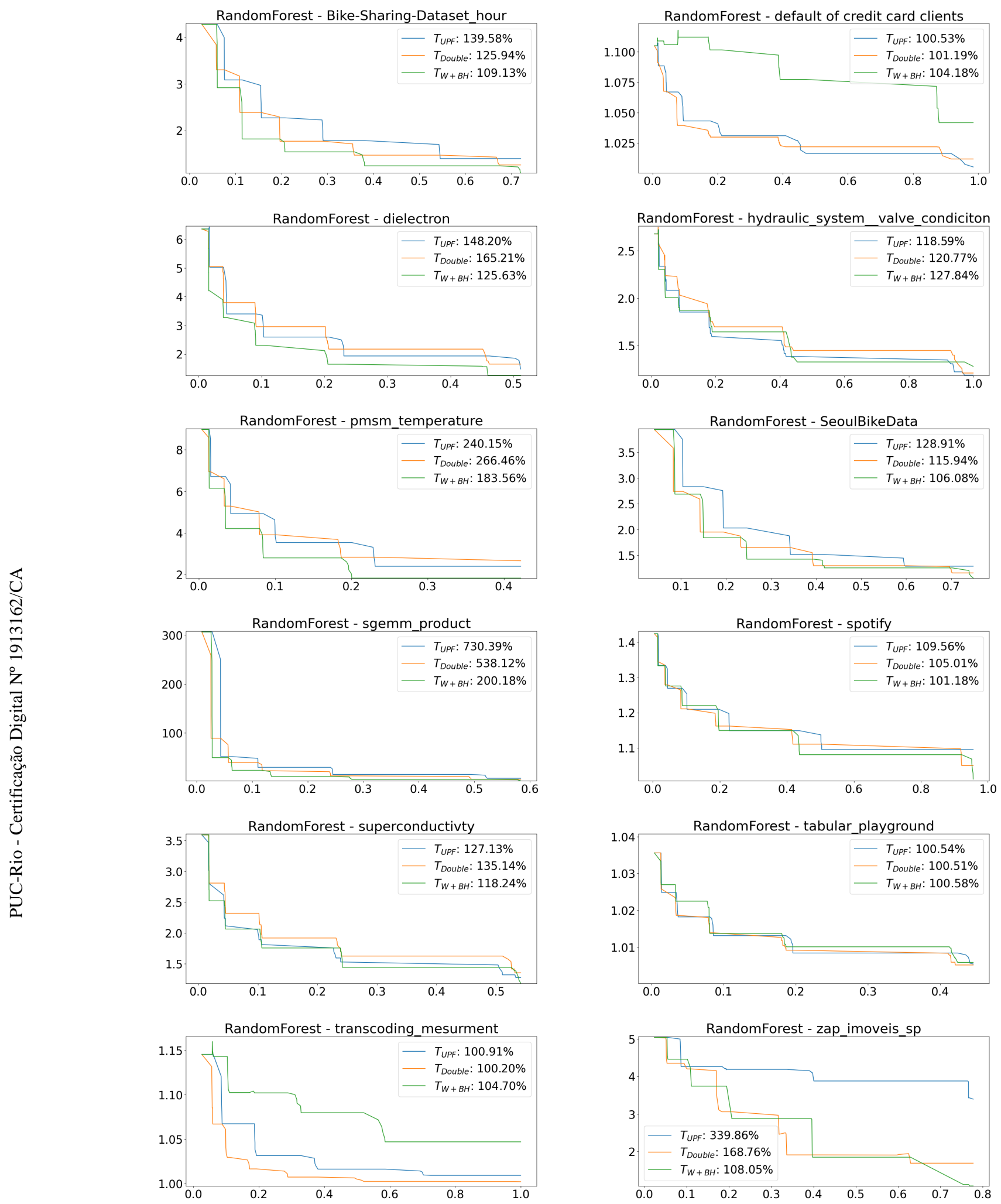

Figura 4.6: Erro médio (eixo vertical) ao longo do tempo (eixo horizontal) no conjunto de teste para as estratégias $T_{U P F}, T_{\text {double }}$ e $T_{W+B H}$, com $L=$ Random Forest. A legenda exibe o erro médio ao fim do experimento. 


\begin{tabular}{l|ccc}
\hline Dataset & $T_{\text {double }}$ & $T_{U P F}$ & $\frac{\text { size }\left(T_{U P F}\right)}{\text { size }\left(T_{\text {Double }}\right)}$ \\
\hline superconductivty & 1.35 & 1.27 & 1 \\
hydraulic system & 1.21 & 1.19 & 1 \\
pmsm temperature & 2.66 & 2.40 & 0.8 \\
zap imoveis sp & 1.69 & 3.40 & 0.5 \\
dielectron & 1.65 & 1.48 & 1 \\
Bike-Sharing-hour & 1.26 & 1.40 & 0.5 \\
sgemm product & 5.38 & 7.30 & 0.5 \\
default of credit card & 1.01 & 1.01 & 1 \\
SeoulBikeData & 1.16 & 1.29 & 0.5 \\
transcoding mesurment & 1.00 & 1.01 & 0.4 \\
spotify & 1.05 & 1.10 & 0.5 \\
tabular playground & 1.01 & 1.01 & 1 \\
\hline Média das médias & 1.70 & 1.90 & - \\
\hline \# erro $T_{U P F}<$ erro $T_{\text {Double }}$ & $5 / 12$ & - \\
\hline
\end{tabular}

Tabela 4.4: Erros dos modelos finais, das estratégias $T_{\text {double }}$ e $T_{U P F}$, para $L=$ Random Forest. Os valores com fundo cinza mostram que estratégia foi melhor em cada caso (cada dataset). A última coluna exibe o tamanho do conj. de treino final de $T_{U P F}$ em relação ao de $T_{\text {double }}$. 


\section{5 \\ Conclusões e Trabalhos Futuros}

\section{1}

\section{Algumas considerações}

Nossa abordagem geral de estratégias (seção 3.1), assume que $L$ não é um algoritmo online. Primeiro, é importante esclarecer que esta premissa não faz parte da definição geral dada ao problema. Segundo, o pseudocódigo pode ser adaptado a este cenário trocando poucas linhas de código (na verdade, apenas a instrução L.FIT $\left.\left(\mathbf{X}_{\text {train }}, \mathbf{Y}_{\text {train }}\right)\right)$. Poderíamos inclusive inserir como input da estratégia uma variável booleana para indicar se $L$ é ou não online (incremental). Em resumo, essa premissa foi assumida apenas para tornar o pseudocódigo mais simples.

A estratégia que adapta $T_{\text {double fazendo um ensemble das hipóteses }}$ produzidas ao longo das interações com $L$ não é uma estratégia válida. Isto porque a hipótese $h^{\prime}$ formada por esse ensemble pode não ser uma das hipóteses que $L$ é capaz de produzir, o que faz parte da exigência do output da estratégia.

Essa exigência foi feita pensando na estratégia como um wrapper de $L$. Do ponto de vista conceitual, pode ser que o usuário, ao escolher $L=$ Árvore de Regressão, queira uma árvore como hipótese (por exemplo, porque o usuário quer obter hipóteses fáceis de compreender). Do ponto de vista prático, pensando na implementação da estratégia numa determinada linguagem de programação, o usuário pode esperar que o objeto retornado pela estratégia tenha uma estrutura de dados específica. Por exemplo, na linguagem Python, as árvores de regressão retornadas pela chamada do método fit de um objeto DecisionTreeRegressor do pacote sklearn são uma estrutura de dados com atributos e métodos específicos ${ }^{1}$, que o usuário pode assumir numa etapa posterior do seu próprio código.

\footnotetext{
${ }^{1}$ Esse objeto retornado por $f i t$ tem os atributos $n_{-}$samples e max_features, cujo significado não é importante nesse contexto
} 


\section{2}

\section{Conclusões}

Nesse trabalho, discutimos estratégias de seleção de exemplos (de treino) em problemas de regressão, cenário em que exemplos rotulados são abundantes e o tempo total de processamento dos dados é escasso. Para formalizar o problema, buscamos uma adaptação do paradigma de Iterative Machine Teaching, proposto em [13].

Nossa estratégia $T_{W+B H}$ seleciona exemplos com base em seu erro de predição e foi avaliada de forma empírica. No conjunto robusto de experimentos que realizamos, $T_{W+B H}$ teve desempenho superior a $T_{\text {double }}$, uma solução natural para o problema em questão, baseada no doubling trick.

Como trabalhos futuros, enxergamos três caminhos: primeiro, tentar obter garantias teóricas (de convergência a uma hipótese-alvo) para as estratégias propostas; segundo, explorar o erro de previsão de exemplo de outras maneiras (como limitar a contribuição do erro ao peso no sorteio, para diminuir a influência de outliers); terceiro, elaborar estratégias que façam seleção de exemplos com base em outras estatísticas que não o erro de previsão, como a incerteza de previsão. 


\section{Referências Bibliográficas}

[1] BOTTOU, L.. On-line learning and stochastic approximations. In: IN ON-LINE LEARNING IN NEURAL NETWORKS, p. 9-42. Cambridge University Press, 1998.

[2] BOTTOU, L.; BOUSQUET, O.. The tradeoffs of large scale learning. In: Platt, J.; Koller, D.; Singer, Y. ; Roweis, S., editors, ADVANCES IN NEURAL INFORMATION PROCESSING SYSTEMS, volumen 20. Curran Associates, Inc., 2008.

[3] BREIMAN, L.; FRIEDMAN, J. H.; OLShEN, R. A. ; STONE, C. J.. Classification and regression trees. Routledge, 2017.

[4] CHANG, C.-C.; LIN, C.-J.. LIBSVM: A library for support vector machines. ACM Transactions on Intelligent Systems and Technology, 2:27:1-27:27, 2011. Software available at http://www.csie.ntu.edu.tw/ c $j$ lin/libsvm.

[5] CHEn, Y.; SINGLA, A.; AODHA, O. M.; PERONA, P. ; YUE, Y.. Understanding the role of adaptivity in machine teaching: The case of version space learners, 2018.

[6] CICAlESE, F.; FILHO, S.; LABER, E. ; MOLINARO, M.. Teaching with limited information on the learner's behaviour. In: III, H. D.; Singh, A., editors, PROCEEDINGS OF THE 37TH INTERNATIONAL CONFERENCE ON MACHINE LEARNING, volumen 119 de Proceedings of Machine Learning Research, p. 2016-2026. PMLR, 13-18 Jul 2020.

[7] COHn, D. A.; GHAHRAMAni, Z. ; JORDAN, M. I.. Active learning with statistical models. CoRR, cs.AI/9603104, 1996.

[8] DASGUPTA, S.; HSU, D.; POULIS, S. ; ZHU, X.. Teaching a black-box learner. In: Chaudhuri, K.; Salakhutdinov, R., editors, PROCEEDINGS OF THE 36TH INTERNATIONAL CONFERENCE ON MACHINE LEARNING, volumen 97 de Proceedings of Machine Learning Research, p. 1547-1555. PMLR, 09-15 Jun 2019. 
[9] DU, J.; LING, C.. Active teaching for inductive learners. In: SDM, 2011.

[10] GOLDMAN, S.; KEARNS, M.. On the complexity of teaching. Journal of Computer and System Sciences, 50(1):20-31, 1995.

[11] HASTIE, T.; TIBSHIRANI, R. ; FRIEDMAN, J.. The Elements of Statistical Learning. Springer Series in Statistics. Springer New York Inc., New York, NY, USA, 2001.

[12] LEWIS, D. D.; CATLETT, J.. Heterogeneous uncertainty sampling for supervised learning. In: Cohen, W. W.; Hirsh, H., editors, ICML, p. 148-156. Morgan Kaufmann, 1994.

[13] LIU, W.; DAI, B.; HUMAYUN, A.; TAY, C.; YU, C.; SMITH, L. B.; REHG, J. M. ; SONG, L.. Iterative machine teaching. In: Precup, D.; Teh, Y. W., editors, PROCEEDINGS OF THE 34TH INTERNATIONAL CONFERENCE ON MACHINE LEARNING, ICML 2017, SYDNEY, NSW, AUSTRALIA, 6-11 AUGUST 2017, volumen 70 de Proceedings of Machine Learning Research, p. 2149-2158. PMLR, 2017.

[14] LIU, W.; DAI, B.; LI, X.; LIU, Z.; REHG, J. M. ; SONG, L.. Towards black-box iterative machine teaching, 2018.

[15] PEDrEgosa, F.; VAROQUAUX, G.; GRAmFOrT, A.; MICHEL, V.; THIRION, B.; GRISEL, O.; BLONDEL, M.; PRETTENHOFER, P.; WEISS, R.; DUBOURG, V.; VANDERPLAS, J.; PASSOS, A.; COURNAPEAU, D.; BRUCHER, M.; PERROT, M. ; DUCHESNAY, E.. Scikitlearn: Machine learning in Python. Journal of Machine Learning Research, 12:2825-2830, 2011.

[16] SETTLES, B.. Active learning literature survey. 2009.

[17] SHALEV-SHWARTZ, S.; BEN-DAVID, S.. Understanding Machine Learning: From Theory to Algorithms. Cambridge University Press, USA, 2014.

[18] VAPNIK, V. N.. Statistical Learning Theory. Wiley-Interscience, 1998.

[19] ZILlES, S.; LANGE, S.; HOLTE, R. ; ZINKEVICH, M.. Models of cooperative teaching and learning. The Journal of Machine Learning Research, 12:349-384, 022011. 


\section{A Experimentos com outros limites de tempo}

Os experimentos da seção 4.6 foram realizados com limite de tempo distinto para cada par ( $L$, dataset). O valor foi o tempo que $L$ leva para treinar com todo o dataset, respeitando o limite superior de 5 minutos.

Este corte (limite superior) foi arbitrado para permitir a análise de sensibilidade dos parâmetros da estratégia $T_{W+B H}$, na seção 4.8 .

Nos gráficos da figura A.1, mostramos que com um limite de tempo distinto, de 1 minuto, as principais conclusões continuam válidas: $T_{W+B H}$ se mostrou superior a $T_{\text {double }}$ em geral, com performance similar à de $T_{\text {double }}$ no caso de Árvores de Regressão e melhor do que a de $T_{\text {double }}$ nos demais casos. 

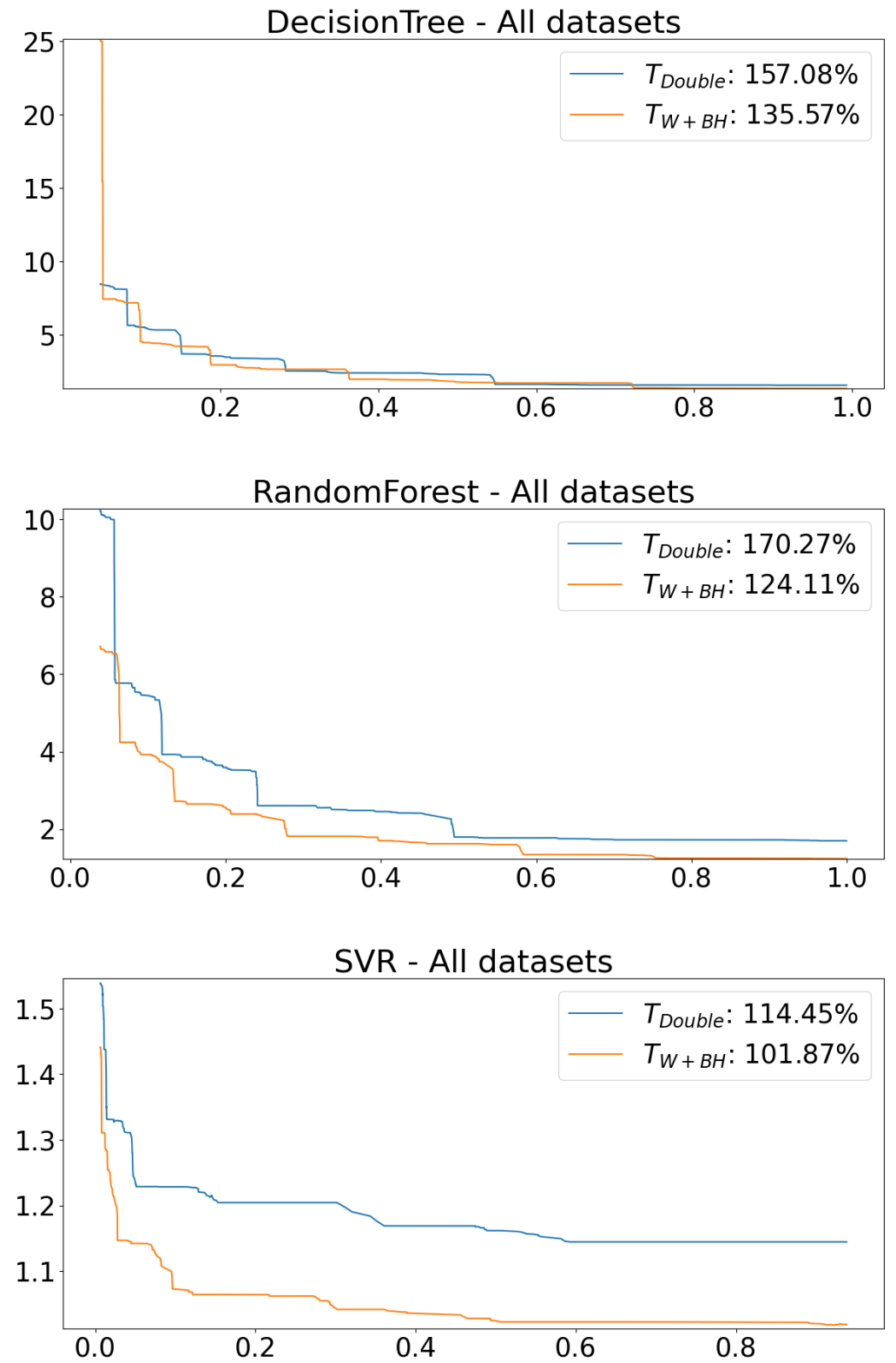

Figura A.1: Erro médio (eixo vertical) ao longo do tempo (eixo horizontal) no conjunto de teste para as estratégias $T_{\text {double }}$ e $T_{W+B H}$ para $L \in\{$ Árvore de Regressão, Random Forest, SVR\}, com o tempo-limite alterado para 1 minuto. A legenda exibe o erro médio ao fim do experimento.

Assim como na seção 4.8, os gráficos (para cada $L$ ), correspondem a médias dos gráficos por dataset. 